\title{
Efficiency of Coupled-Cluster Singles and Doubles on Modern Stream Processing Architectures
}

B. Scott Fales, ${ }^{1,2}$ Ethan R. Curtis, ${ }^{1,2}$ K. Grace Johnson, ${ }^{1,2}$

Dean Lahana, ${ }^{1,2}$ Stefan Seritan, ${ }^{1,2}$ Yuanheng Wang, ${ }^{1,2}$ Hayley

Weir, ${ }^{1,2}$ Todd J. Martínez, ${ }^{1,2}$ and Edward G. Hohenstein ${ }^{1,2, *}$

${ }^{1}$ Department of Chemistry and the PULSE Institute,

Stanford University, Stanford, CA 94305

${ }^{2} S L A C$ National Accelerator Laboratory,

2575 Sand Hill Road, Menlo Park, CA 94025 


\section{THEORY OVERVIEW}

In this work, we employ the closed-shell spin-adapted formalism of Koch et al. ${ }^{1}$ and use the naming conventions outlined in Molecular Electronic Structure Theory. ${ }^{2}$ Below, we briefly summarize the quantities needed to perform a CCSD computation and reproduce the spin-adapted amplitude equations. We follow the convention that $i, j, k, \ldots$ index active occupied orbitals, $a, b, c, \ldots$ index virtual orbitals, $p, q, r, \ldots$ index general molecular orbitals $(\mathrm{MO}), \mu, \nu, \lambda, \ldots$ index atomic orbitals $(\mathrm{AO})$ and $A, B, C, \ldots$ index Cholesky vectors.

\section{A. Notation and Definitions}

To form matrix elements of the $t_{1}$-transformed Hamiltonian, the particle and hole modified MO coefficient matrices are used $\left(\tilde{C}^{(p)}\right.$ and $\left.\tilde{C}^{(h)}\right)$.

$$
\begin{gathered}
\tilde{C}_{\mu i}^{(p)}=C_{\mu i} \\
\tilde{C}_{\mu a}^{(p)}=C_{\mu a}-t_{i}^{a} C_{\mu i}, \\
\tilde{C}_{\mu i}^{(h)}=C_{\mu i}+t_{i}^{a} C_{\mu a} \\
\tilde{C}_{\mu i}^{(h)}=C_{\mu a}
\end{gathered}
$$

The Cholesky vectors in the $\mathrm{AO}$ or $\mathrm{MO}$ basis are denoted as $B$, throughout, while the Cholesky vectors in the modified MO basis (i.e. including contributions from singles amplitudes) are denoted $\tilde{B}$.

$$
\begin{gathered}
(\mu \nu \mid \rho \sigma) \approx \sum_{A} B_{\mu \nu}^{A} B_{\rho \sigma}^{A} \\
B_{p q}^{A}=\sum_{\mu \nu} C_{\mu p} C_{\mu q} B_{\mu \nu}^{A} \\
\tilde{B}_{p q}^{A}=\sum_{\mu \nu} \tilde{C}_{\mu p}^{(p)} \tilde{C}_{\mu q}^{(h)} B_{\mu \nu}^{A} \\
(p q \mid r s) \approx \sum_{A} B_{p q}^{A} B_{r s}^{A} \\
\tilde{G}_{p r q s}=\tilde{g}_{p q r s} \approx \sum_{A} \tilde{B}_{p q}^{A} \tilde{B}_{r s}^{A}
\end{gathered}
$$




$$
\tilde{L}_{p q r s}=2 \tilde{g}_{p q r s}-\tilde{g}_{p s r q}
$$

The modified Fock operator is formed from one- and two-electron operators in the modified MO basis.

$$
\begin{gathered}
\tilde{F}_{p q}=\sum_{\mu \nu}\left(\tilde{C}_{\mu p}^{(p)} \tilde{C}_{\mu q}^{(h)} \tilde{h}_{\mu \nu}\right)+\sum_{i}\left(2 \tilde{g}_{p q i i}-\tilde{g}_{p i i q}\right) \\
u_{i j a b}=2 t_{i j a b}-t_{i j b a}
\end{gathered}
$$

\section{B. CCSD singles residual}

The singles amplitudes, $t_{i}^{a}$, are defined by the iterative solution of the following nonlinear

equations (coupled to the doubles amplitudes). The details of the spin adaptation can be found in Koch et al. ${ }^{1}$ and in Molecular Electronic Structure Theory. ${ }^{2}$

$$
\begin{gathered}
\left\langle\Phi_{i}^{a}\left|e^{-\hat{T}} \hat{H} e^{\hat{T}}\right| \Phi_{0}\right\rangle=\Omega_{a i}=\Omega_{a i}^{\mathrm{A} 1}+\Omega_{a i}^{\mathrm{B} 1}+\Omega_{a i}^{\mathrm{C} 1}+\Omega_{a i}^{\mathrm{D} 1} \\
\Omega_{a i}^{\mathrm{A} 1}=2 \sum_{k c d} u_{k i c d} \tilde{g}_{a d k c} \\
\Omega_{a i}^{\mathrm{B} 1}=-2 \sum_{k l c} u_{k l a c} \tilde{g}_{k i l c} \\
\Omega_{a i}^{\mathrm{C} 1}=2 \sum_{k c} u_{i k a c} \tilde{F}_{k c} \\
\Omega_{a i}^{\mathrm{D} 1}=2 \tilde{F}_{a i}
\end{gathered}
$$

\section{CCSD doubles residual}

The doubles amplitudes, $t_{i j}^{a b}$, are defined by the iterative solution of the following nonlinear equations (coupled to the singles amplitudes). The details of the spin adaptation can be found in Koch et al. ${ }^{1}$ and in Molecular Electronic Structure Theory. ${ }^{2}$

$$
\begin{aligned}
\left\langle\Phi_{i j}^{a b}\left|e^{-\hat{T}} \hat{H} e^{\hat{T}}\right| \Phi_{0}\right\rangle & =\Omega_{a i b j} \\
& =\Omega_{a i b j}^{\mathrm{A} 2}+\Omega_{a i b j}^{\mathrm{B} 2}+\Omega_{a i b j}^{\mathrm{F} 2}+P(a i, b j)\left(\Omega_{a i b j}^{\mathrm{C} 2}+2 \Omega_{a j b i}^{\mathrm{C} 2}+\Omega_{a i b j}^{\mathrm{D} 2}+\Omega_{a i b j}^{\mathrm{E} 2}\right)
\end{aligned}
$$




$$
\begin{gathered}
\Omega_{a i b j}^{\mathrm{A} 2}=2 \sum_{k l} t_{k l a b}\left(\tilde{g}_{k i l j}+\sum_{c d} t_{i j c d} \tilde{g}_{k c l d}\right) \\
\Omega_{a i b j}^{\mathrm{B} 2}=2 \sum_{c d} t_{i j c d} \tilde{g}_{a c b d} \\
\Omega_{a i b j}^{\mathrm{C} 2}=-\sum_{k c} t_{k j b c}\left(\tilde{g}_{k i a c}-\frac{1}{2} \sum_{l d} t_{l i a d} \tilde{g}_{k d l c}\right) \\
\Omega_{a i b j}^{\mathrm{D} 2}=\sum_{k c} u_{j k b c}\left(\tilde{L}_{a i k c}+\frac{1}{2} \sum_{l d} u_{i l a d} \tilde{L}_{l d k c}\right) \\
\Omega_{a i b j}^{\mathrm{E} 2}=2 \sum_{c} t_{i j a c}\left(\tilde{F}_{b c}-\sum_{k l d} u_{k l b d} \tilde{g}_{l d k c}\right)-2 \sum_{k} t_{i k a b}\left(\tilde{F}_{k j}+\sum_{l c d} u_{l j c d} \tilde{g}_{k d l c}\right) \\
\Omega_{a i b j}^{\mathrm{F} 2}=2 \tilde{g}_{a i b j}
\end{gathered}
$$

\section{ALGORITHM FOR GPU ACCELERATED FORMATION OF THE CCSD RESIDUAL}

In this section, we provide pseudocode for our GPU-accelerated CCSD algorithm. Before the iterations begin, the $\tilde{g}_{i a j b}$ two-electron integrals are formed, since they do not depend on the $t_{1}$ amplitudes. Each iteration, the Cholesky vectors are transformed from the AO to modifed MO basis and the contraction $T_{i a}^{A}=\sum_{j b} u_{i a j b} \tilde{B}_{j b}^{A}$ is performed. For reference, the operation count for each of these contributions is listed in Table S1. However, these

operation counts reflect only the best case, where the limited memory on the GPU does not necessitate redundant computation. 
TABLE S1: Nominal operation counts for each of the steps in a CCSD iteration. Counts are given in terms of multiply-add operations and include only matrix-matrix and matrix-vector multiplications.

\begin{tabular}{lcc}
\hline \hline Term & Algorithm & Operation count \\
\hline MO integrals & 1 & $N_{m o} N_{a o}^{2} N_{c d}+N_{m o}^{2} N_{a o} N_{c d}$ \\
$T_{i a}^{A}$ & 2 & $N_{o}^{2} N_{v}^{2} N_{c d}$ \\
$\tilde{g}_{i a j b}$ & 3 & $N_{o}^{2} N_{v}^{2} N_{c d}$ \\
$\Omega^{\mathrm{A} 1}$ & 4 & $N_{o} N_{v}^{2} N_{c d}$ \\
$\Omega^{\mathrm{B} 1}$ & 4 & $N_{o}^{2} N_{v} N_{c d}$ \\
$\Omega^{\mathrm{C} 1}$ & 9 & $N_{o}^{2} N_{v}^{2}$ \\
$\Omega^{\mathrm{A} 2}$ & 5 & $N_{o}^{4} N_{c d}+\frac{3}{2} N_{o}^{4} N_{v}^{2}$ \\
$\Omega^{\mathrm{B} 2}$ & 6 & $\frac{1}{2} N_{v}^{4} N_{c d}+\frac{1}{4} N_{o}^{2} N_{v}^{4}$ \\
$\Omega^{\mathrm{C} 2}$ & 7 & $N_{o}^{2} N_{v}^{2} N_{c d}+2 N_{o}^{3} N_{v}^{3}$ \\
$\Omega^{\mathrm{D} 2}$ & 8 & $2 N_{o}^{2} N_{v}^{2} N_{c d}+2 N_{o}^{3} N_{v}^{3}$ \\
$\Omega^{\mathrm{E} 2}$ & 10 & $N_{o}^{2} N_{v} N_{c d}+N_{o} N_{v}^{2} N_{c d}+N_{o}^{3} N_{v}^{2}+N_{o}^{2} N_{v}^{3}$ \\
$\Omega^{\mathrm{F} 2}$ & 8 & $N_{o}^{2} N_{v}^{2} N_{c d}$ \\
\hline \hline
\end{tabular}

$N_{a o}$ : Number of atomic orbitals. $N_{m o}$ : Number of molecular orbitals. $N_{o}$ : Number of occupied orbitals. $N_{v}$ : Number of virtual orbitals. $N_{c d}$ : Length of Cholesky decomposition.

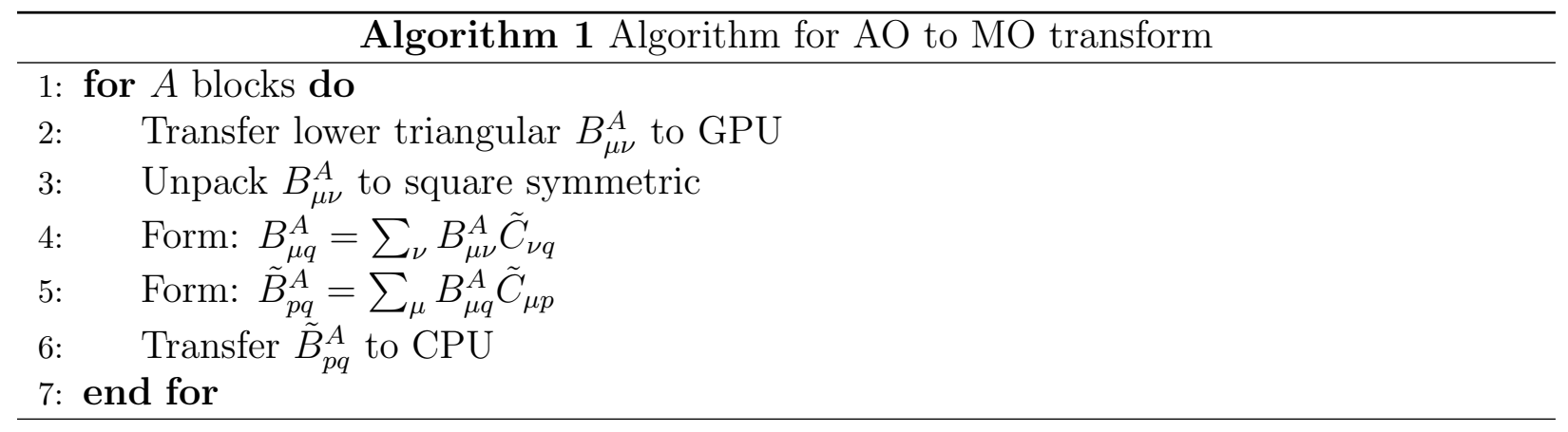

\section{Algorithm 2 Algorithm for $T_{i a}^{A}$}

$\begin{array}{ll}\text { 1: } & \text { for } i \text { blocks do } \\ 2: & \text { for } A \text { blocks do } \\ 3: & \text { Transfer } t_{i j a b} \text { and } \tilde{B}_{j b}^{A} \text { to GPU } \\ \text { 4: } & \text { Antisymmetrize: } u_{i a j b} \leftarrow 2 t_{i j a b}-t_{i j b a} \\ 5: & \text { Form: } T_{i a}^{A}=\sum_{j b} u_{i a j b} \tilde{B}_{j b}^{A} \\ 6: & \text { Transfer: } T_{i a}^{A} \text { to CPU } \\ \text { 7: } & \text { end for } \\ \text { 8: end for }\end{array}$


Algorithm 3 Algorithm for $\tilde{g}_{i a j b}$
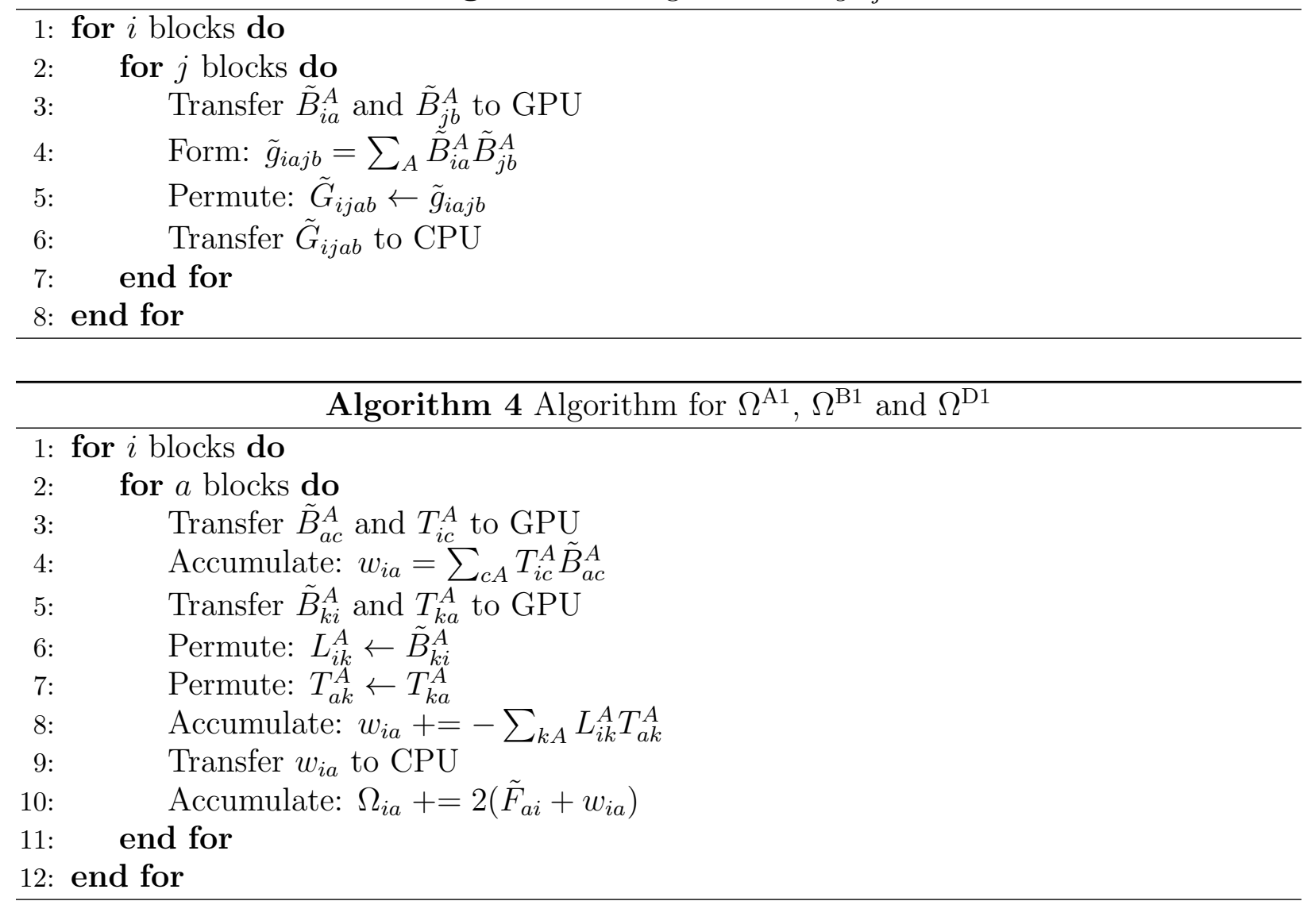

Algorithm 5 Algorithm for $\Omega^{\mathrm{A} 2}$ 


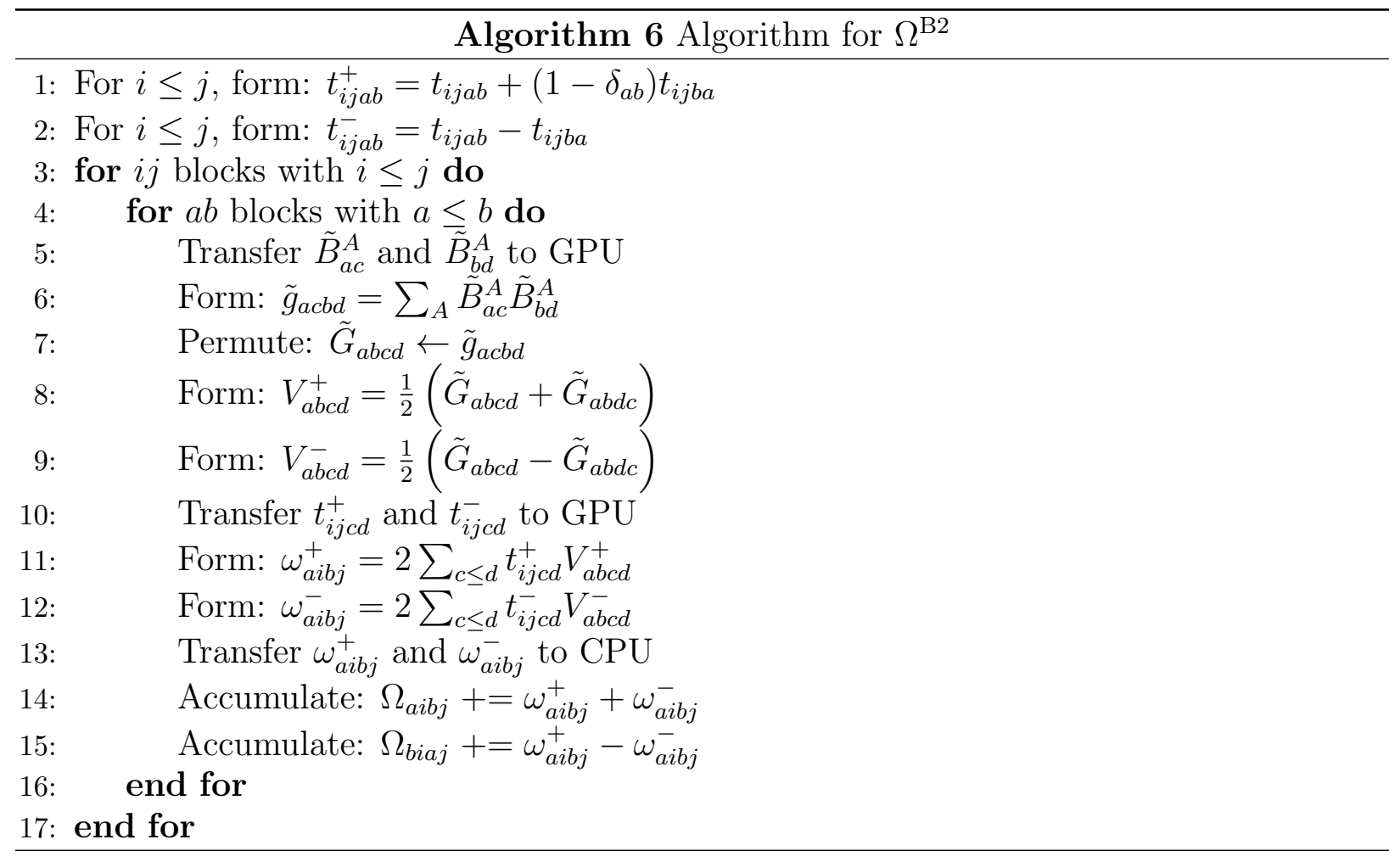

\section{Algorithm 7 Algorithm for $\Omega^{\mathrm{C} 2}$}

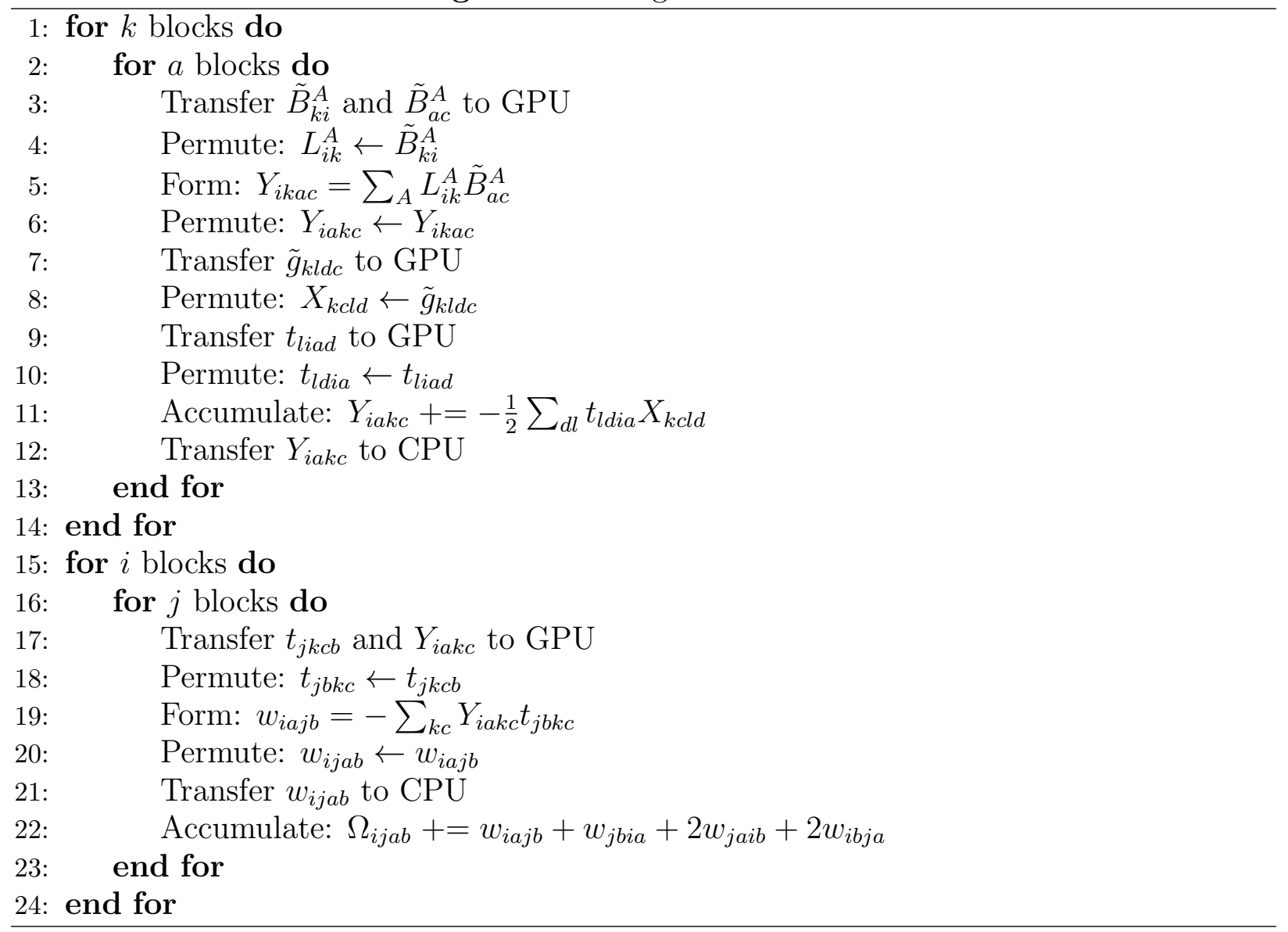




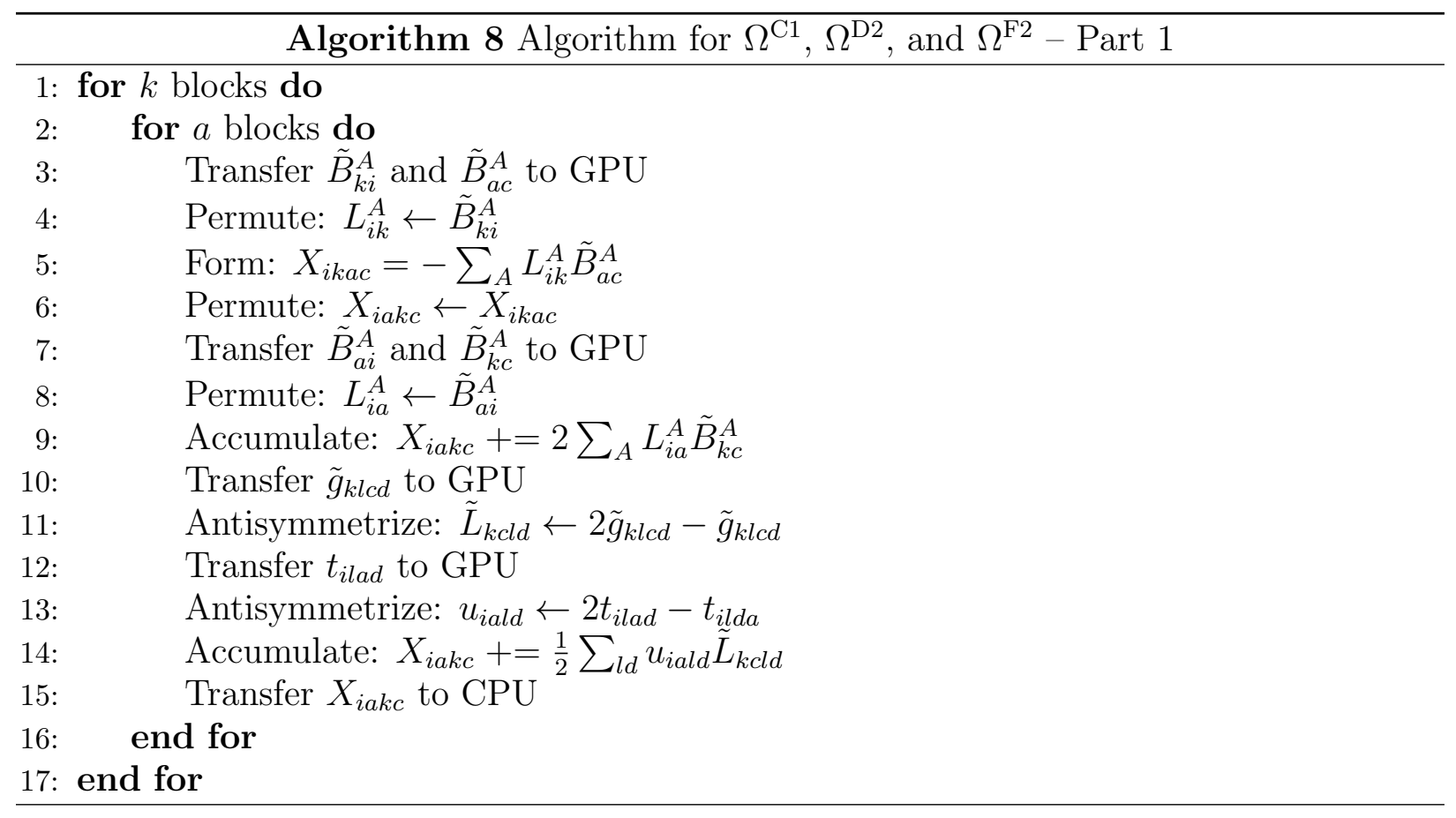




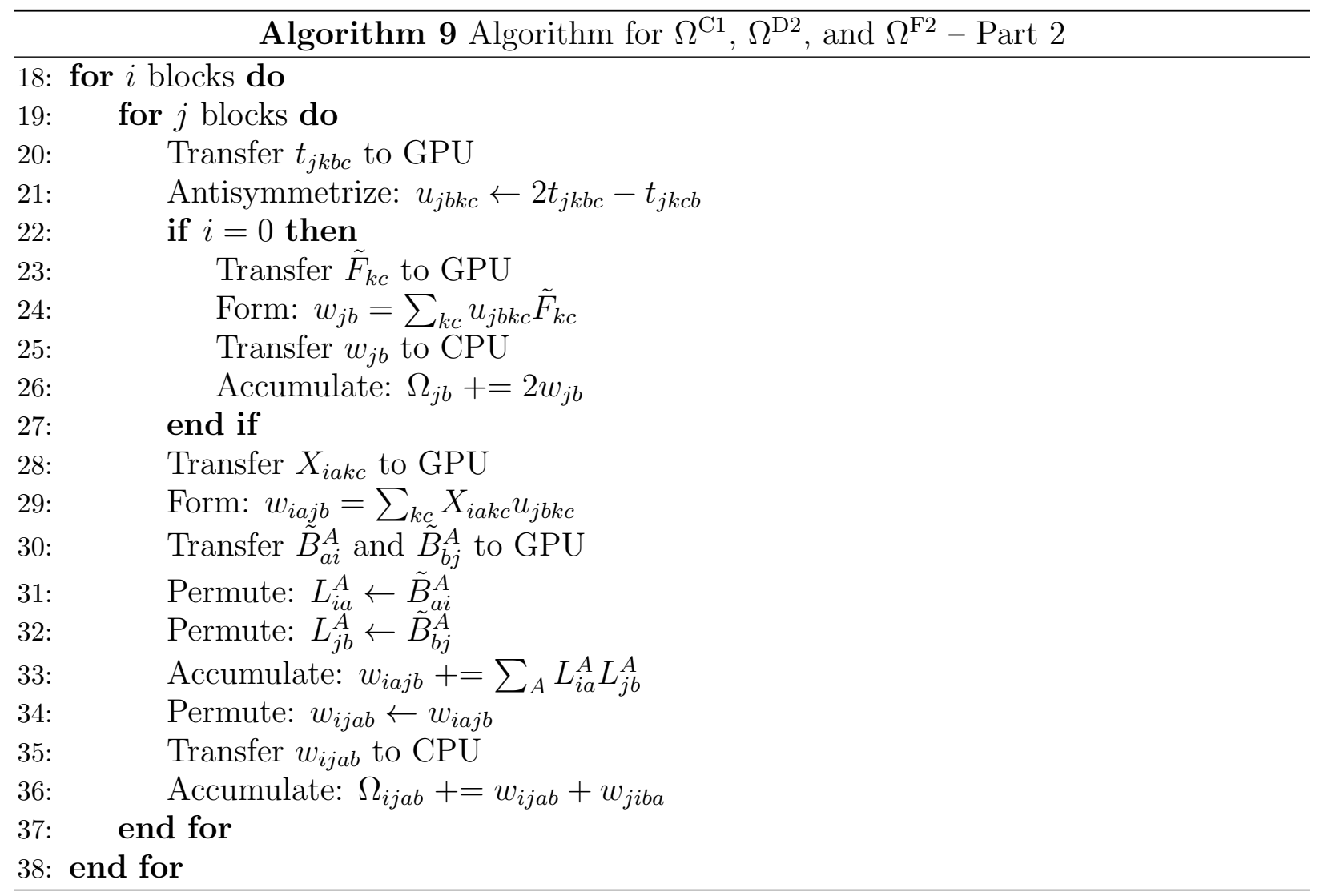


Algorithm 10 Algorithm for $\Omega^{\mathrm{E} 2}$
1: for $j$ blocks do
2: $\quad$ for $k$ blocks do
3: $\quad$ Transfer $\tilde{B}_{k c}^{A}$ and $T_{j c}^{A}$ to GPU
4: $\quad$ Form: $Y_{k j}=\sum_{c A} \tilde{B}_{k c}^{A} T_{j c}^{A}$
5: $\quad$ Transfer $Y_{k j}$ to CPU
6: $\quad$ Form: $X_{j k}=F_{k j}+Y_{k j}$
7: $\quad$ end for
8: end for
9: for $b$ blocks do
10: $\quad$ for $c$ blocks do
11: $\quad$ Transfer $\tilde{B}_{k c}^{A}$ and $T_{k b}^{A}$ to GPU
12: $\quad$ Permute: $L_{c k}^{A} \leftarrow \tilde{B}_{k c}^{A}$
13: $\quad$ Permute: $T_{b k}^{A} \leftarrow T_{k b}^{A}$
14: $\quad$ Form: $Y_{b c}=\sum_{k A} T_{b k}^{A} L_{c k}^{A}$
15: $\quad$ Transfer $Y_{b c}$ to CPU
16: $\quad$ Form: $X_{b c}=F_{b c}-Y_{b c}$
17: $\quad$ end for
18: end for
19: for $j$ blocks do
20: $\quad$ for $a$ blocks do
21: $\quad$ Transfer $t_{i j a b}, X_{j k}$, and $X_{b c}$ to GPU
22: $\quad$ Accumulate: $w_{i j a b}+=2 \sum_{c} t_{i j a c} X_{b c}$
23: $\quad$ Accumulate: $w_{i j a b}+=-2 \sum_{k} t_{i k a b} X_{j k}$
24: $\quad$ Transfer $w_{i j a b}$ to $\mathrm{CPU}$
25: $\quad$ Accumulate: $\Omega_{i j a b}+=w_{i j a b}+w_{j i b a}$
26: end for
27: end for 


\section{DETAILED ANALYSIS OF COMPUTATIONAL EFFICIENCY}

In Tables S2 and S3, we report a decomposition of the timings of our CCSD program for one iteration on a $\left(\mathrm{H}_{2} \mathrm{O}\right)_{20}$ cluster in the TZVP basis set. In these computations, the frozen core approximation was applied to the oxygen $1 s$ orbitals and a Cholesky decomposition threshold of $10^{-6} \mathrm{E}_{\mathrm{h}}$ was used. To avoid complicating the analysis of the timings, only one GPU and one CPU core were used; we present timings for both the NVIDIA GTX $1080 \mathrm{Ti}$ GPU (Table S2) and NVIDIA V100 GPU (Table S3).

In addition to timings, we present an analysis of the performance in terms of floating point operations per second (FLOPs) as well as memory transfer rates between the GPU and CPU. What we report as "Memory time" is dominated by memory transfer between the CPU and GPU, but also contains allocation/deallocation of memory as well as initialization of arrays. "CPU time" is any work done exclusively on the CPU that is interspersed between calls to the GPU (re-ordering an array, for example). The "GPU kernel time" consists of the time spent in the CUDA kernels we wrote to manipulate arrays on the GPU. The "GPU DGEMM time" contains the time spent in the cuBLAS matrix multiplication functions on the GPU. "Total time" is the sum of the previously mentioned catagories. In some cases, CPU-based pre- or post-processing of arrays is needed, these times are included separately in "CPU pre/post". Finally, note that there are some relatively inexpensive operations performed on the CPU (such as computing the norm of the residual), which account for the small difference between the "Total iteration time" and the sum of the reported components; this contribution is accumulated in "Miscellaneous time". The apparent FLOP rates we report inlcude only the rate-limiting matrix-matrix multiplications and, therefore, slightly underestimate the true FLOP rate.

Finally, a note on the specific hardware used for these timings. The V100 GPU used here has a base clock speed of $1.44 \mathrm{GHz}$ (resulting in a theoretical peak of 7.37 TFLOPs). The GTX 1080 Ti has a base clock of 1.48 GHz (332 GFLOPs peak), but has a max boost clock of $1.91 \mathrm{GHz}$. It is the boost clock that accounts for the, perhaps, higher then expected FLOP rates. 
TABLE S2: Timings of one CCSD/TZVP iteration on the $\left(\mathrm{H}_{2} \mathrm{O}\right)_{20}$ cluster using 1 NVIDIA GTX 1080 Ti GPU.

\begin{tabular}{|c|c|c|c|c|}
\hline \multicolumn{2}{|c|}{ Contribution } & Time $(\mathrm{s})$ & Perfor & rmance \\
\hline \multirow[t]{5}{*}{$\tilde{g}_{i a j b}$} & Memory time & 5.15 & 3.91 & $\mathrm{~Gb} / \mathrm{s}$ \\
\hline & CPU time & 1.38 & & \\
\hline & GPU kernel time & 0.19 & & \\
\hline & GPU DGEMM time & 41.75 & 389.93 & GFlops \\
\hline & Total time & 48.46 & 335.88 & GFlops \\
\hline \multirow[t]{5}{*}{ MO Integrals } & Memory time & 5.78 & 3.41 & $\mathrm{~Gb} / \mathrm{s}$ \\
\hline & CPU time & 2.23 & & \\
\hline & GPU kernel time & 0.07 & & \\
\hline & GPU DGEMM time & 13.78 & 329.09 & GFlops \\
\hline & Total time & 21.90 & 207.17 & GFlops \\
\hline \multirow[t]{4}{*}{$T_{i a}^{A}$} & Memory time & 4.09 & 4.92 & $\mathrm{~Gb} / \mathrm{s}$ \\
\hline & GPU kernel time & 0.00 & & \\
\hline & GPU DGEMM time & 43.04 & 378.24 & GFlops \\
\hline & Total time & 47.32 & 343.99 & GFlops \\
\hline \multirow[t]{5}{*}{$\Omega^{\mathrm{A} 1, \mathrm{~B} 1, \mathrm{D} 1}$} & Memory time & 2.09 & 7.34 & $\mathrm{~Gb} / \mathrm{s}$ \\
\hline & CPU time & 0.00 & & \\
\hline & GPU kernel time & 0.04 & & \\
\hline & GPU DGEMM time & 1.22 & 192.72 & GFlops \\
\hline & Total time & 3.35 & 70.12 & GFlops \\
\hline \multirow[t]{5}{*}{$\Omega^{\mathrm{A} 2}$} & Memory time & 21.04 & 6.39 & $\mathrm{~Gb} / \mathrm{s}$ \\
\hline & CPU time & 1.06 & & \\
\hline & GPU kernel time & 0.10 & & \\
\hline & GPU DGEMM time & 91.45 & 369.04 & GFlops \\
\hline & Total time & 113.66 & 296.94 & GFlops \\
\hline \multirow[t]{6}{*}{$\Omega^{\mathrm{B} 2}$} & Memory time & 117.76 & 6.61 & $\mathrm{~Gb} / \mathrm{s}$ \\
\hline & CPU time & 0.00 & & \\
\hline & GPU kernel time & 8.22 & & \\
\hline & GPU DGEMM time & 1680.31 & 372.51 & GFlops \\
\hline & Total time & 1809.53 & 345.91 & GFlops \\
\hline & CPU pre/post & 4.71 & & \\
\hline \multirow[t]{6}{*}{$\Omega^{\mathrm{C} 2}$} & Memory time & 45.17 & 6.44 & $\mathrm{~Gb} / \mathrm{s}$ \\
\hline & CPU time & 3.77 & & \\
\hline & GPU kernel time & 2.82 & & \\
\hline & GPU DGEMM time & 804.98 & 377.95 & GFlops \\
\hline & Total time & 856.74 & 355.12 & GFlops \\
\hline & CPU pre/post & 10.38 & & \\
\hline \multirow[t]{6}{*}{$\Omega^{\mathrm{C} 1, \mathrm{D} 2, \mathrm{~F} 2}$} & Memory time & 54.53 & 5.79 & $\mathrm{~Gb} / \mathrm{s}$ \\
\hline & CPU time & 4.63 & & \\
\hline & GPU kernel time & 3.55 & & \\
\hline & GPU DGEMM time & 891.72 & 377.69 & GFlops \\
\hline & Total time & 954.48 & 352.86 & GFlops \\
\hline & CPU pre/post & 9.56 & & \\
\hline \multirow[t]{5}{*}{$\Omega^{\mathrm{E} 2}$} & Memory time & 8.45 & 4.02 & $\mathrm{~Gb} / \mathrm{s}$ \\
\hline & CPU time & 1.97 & & \\
\hline & GPU kernel time & 0.05 & & \\
\hline & GPU DGEMM time & 7.82 & 295.59 & GFlops \\
\hline & Total time & 18.29 & 126.36 & GFlops \\
\hline \multicolumn{2}{|l|}{ Total memory time } & 258.92 & 6.67 & $\%$ \\
\hline \multicolumn{2}{|l|}{ Total CPU time } & 13.65 & 0.35 & $\%$ \\
\hline \multicolumn{2}{|l|}{ Total GPU kernal time } & 14.86 & 0.38 & $\%$ \\
\hline \multicolumn{2}{|l|}{ Total GPU DGEMM time } & 3534.32 & 91.08 & $\%$ \\
\hline \multicolumn{2}{|l|}{ Total CPU pre/post time } & 24.65 & 0.64 & $\%$ \\
\hline \multicolumn{2}{|l|}{ Miscellaneous time } & 34.24 & 0.88 & $\%$ \\
\hline \multicolumn{2}{|l|}{ Total iteration time } & 3880.64 & 341.20 & GFlops \\
\hline
\end{tabular}


TABLE S3: Timings of one CCSD/TZVP iteration on the $\left(\mathrm{H}_{2} \mathrm{O}\right)_{20}$ cluster using 1 NVIDIA V100 GPU.

\begin{tabular}{|c|c|c|c|c|}
\hline \multicolumn{2}{|c|}{ Contribution } & \multirow{2}{*}{$\begin{array}{r}\text { Time }(\mathrm{s}) \\
8.23\end{array}$} & \multicolumn{2}{|c|}{ Performance } \\
\hline \multirow[t]{5}{*}{$\tilde{g}_{i a j b}$} & Memory time & & 2.06 & $\mathrm{~Gb} / \mathrm{s}$ \\
\hline & CPU time & 4.48 & & \\
\hline & GPU kernel time & 0.09 & & \\
\hline & GPU DGEMM time & 4.35 & 3744.58 & GFlops \\
\hline & Total time & 17.15 & 949.10 & GFlops \\
\hline \multirow[t]{5}{*}{ MO Integrals } & Memory time & 8.60 & 2.29 & $\mathrm{~Gb} / \mathrm{s}$ \\
\hline & CPU time & 5.35 & & \\
\hline & GPU kernel time & 0.05 & & \\
\hline & GPU DGEMM time & 1.54 & 2953.90 & GFlops \\
\hline & Total time & 15.54 & 291.92 & GFlops \\
\hline \multirow{4}{*}{$T_{i a}^{A}$} & Memory time & 5.89 & 2.88 & $\mathrm{~Gb} / \mathrm{s}$ \\
\hline & GPU kernel time & 0.00 & & \\
\hline & GPU DGEMM time & 3.43 & 4750.55 & GFlops \\
\hline & Total time & 9.41 & 1729.90 & GFlops \\
\hline \multirow[t]{5}{*}{$\Omega^{\mathrm{A} 1, \mathrm{~B} 1, \mathrm{D} 1}$} & Memory time & 3.84 & $=3.53$ & $\mathrm{~Gb} / \mathrm{s}$ \\
\hline & CPU time & 0.00 & & \\
\hline & GPU kernel time & 0.02 & & \\
\hline & GPU DGEMM time & 0.26 & 918.34 & GFlops \\
\hline & Total time & 4.12 & 57.00 & GFlops \\
\hline \multirow[t]{5}{*}{$\Omega^{\mathrm{A} 2}$} & Memory time & 17.49 & 3.65 & $\mathrm{~Gb} / \mathrm{s}$ \\
\hline & CPU time & 2.30 & & \\
\hline & GPU kernel time & 0.02 & & \\
\hline & GPU DGEMM time & 9.61 & 3511.99 & GFlops \\
\hline & Total time & 29.43 & 1146.76 & GFlops \\
\hline \multirow[t]{6}{*}{$\Omega^{\mathrm{B} 2}$} & Memory time & 82.11 & 3.36 & $\mathrm{~Gb} / \mathrm{s}$ \\
\hline & CPU time & 0.00 & & \\
\hline & GPU kernel time & 3.61 & & \\
\hline & GPU DGEMM time & 190.02 & 3495.70 & GFlops \\
\hline & Total time & 284.40 & 2335.59 & GFlops \\
\hline & CPU pre/post & 6.63 & & \\
\hline \multirow[t]{6}{*}{$\Omega^{\mathrm{C} 2}$} & Memory time & 49.19 & 3.04 & $\mathrm{~Gb} / \mathrm{s}$ \\
\hline & CPU time & 11.35 & & \\
\hline & GPU kernel time & 1.13 & & \\
\hline & GPU DGEMM time & 84.53 & 3599.29 & GFlops \\
\hline & Total time & 146.20 & 2081.02 & GFlops \\
\hline & CPU pre/post & 19.22 & & \\
\hline \multirow[t]{6}{*}{$\Omega^{\mathrm{C} 1, \mathrm{D} 2, \mathrm{~F} 2}$} & Memory time & 57.02 & 2.84 & $\mathrm{~Gb} / \mathrm{s}$ \\
\hline & CPU time & 12.86 & & \\
\hline & GPU kernel time & 1.00 & & \\
\hline & GPU DGEMM time & 101.83 & 3307.40 & GFlops \\
\hline & Total time & 172.75 & 1949.57 & GFlops \\
\hline & CPU pre/post & 18.06 & & \\
\hline \multirow[t]{5}{*}{$\Omega^{\mathrm{E} 2}$} & Memory time & 14.68 & 2.31 & $\mathrm{~Gb} / \mathrm{s}$ \\
\hline & CPU time & 4.52 & & \\
\hline & GPU kernel time & 0.03 & & \\
\hline & GPU DGEMM time & 0.67 & 3425.29 & GFlops \\
\hline & Total time & 19.91 & 116.10 & GFlops \\
\hline Total memory time & & 238.83 & 31.84 & $\%$ \\
\hline Total CPU time & & 36.38 & 4.85 & $\%$ \\
\hline Total GPU kernal time & & 5.87 & 0.78 & $\%$ \\
\hline Total GPU DGEMM time & & 391.88 & 52.25 & $\%$ \\
\hline Total CPU pre/post time & & 43.90 & 5.85 & $\%$ \\
\hline Miscellaneous time & & 33.16 & 4.42 & $\%$ \\
\hline Total iteration time & & 750.03 & 1816.45 & GFlops \\
\hline
\end{tabular}




\section{CARTESIAN COORDINATES OF MOLECULAR GEOMETRIES USED IN THIS WORK}

The cartesian coordinates (in $\AA$ ) for all of the molecular systems considered in this work are provided. The numbers of basis functions, orbitals, and the length of the Cholesky decomposition are provided in Table S4. The geometries for the methylated uracil dimer $\left((\mathrm{mU})_{2} \cdot \mathrm{H}_{2} \mathrm{O}\right)$ and adenine-thymine stack (AATT) were taken from Epifanovsky et al.; ${ }^{3}$ the geometry for beta carotene was taken from $\mathrm{Hu}$ et al. ${ }^{4}$ For all molecules, the Cholesky decomposition was set to be $10^{-4}$ or to the length of the analogous density fitting basis (for timings comparisions against other software packages). The exception to this is AATT, where a threshold of $10^{-2}$ was used to be consistent with Epifanovsky et al. ${ }^{3}$

TABLE S4: Details of molecular systems considered in this work.

\begin{tabular}{|c|c|c|c|c|c|}
\hline Molecule & Basis set & Active occupied Orbitals & Virtual orbitals & Basis functions & Cholesky vectors \\
\hline$\left(\mathrm{H}_{2} \mathrm{O}\right)_{5}$ & TZVP & 20 & 130 & 155 & 657 \\
\hline$\left(\mathrm{H}_{2} \mathrm{O}\right)_{10}$ & cc-pVDZ & 40 & 190 & 240 & 920 \\
\hline$\left(\mathrm{H}_{2} \mathrm{O}\right)_{10}$ & TZVP & 40 & 260 & 310 & 1314 \\
\hline$\left(\mathrm{H}_{2} \mathrm{O}\right)_{15}$ & TZVP & 60 & 390 & 465 & 1970 \\
\hline$\left(\mathrm{H}_{2} \mathrm{O}\right)_{15}$ & aug-cc-pVDZ & 60 & 540 & 615 & 2489 \\
\hline$\left(\mathrm{H}_{2} \mathrm{O}\right)_{19}$ & TZVP & 76 & 494 & 589 & 2499 \\
\hline$\left(\mathrm{H}_{2} \mathrm{O}\right)_{19}$ & aug-cc-pVDZ & 76 & 684 & 779 & 3163 \\
\hline$\left(\mathrm{H}_{2} \mathrm{O}\right)_{20}$ & TZVP & 80 & 520 & 620 & 2617 \\
\hline$\left(\mathrm{H}_{2} \mathrm{O}\right)_{24}$ & TZVP & 96 & 624 & 744 & 3159 \\
\hline$\left(\mathrm{H}_{2} \mathrm{O}\right)_{24}$ & aug-cc-pVDZ & 96 & 864 & 984 & 4019 \\
\hline$\left(\mathrm{H}_{2} \mathrm{O}\right)_{25}$ & TZVP & 100 & 650 & 775 & 3296 \\
\hline$\left(\mathrm{H}_{2} \mathrm{O}\right)_{30}$ & TZVP & 120 & 780 & 930 & 3943 \\
\hline$\left(\mathrm{H}_{2} \mathrm{O}\right)_{30}$ & aug-cc-pVDZ & 120 & 1080 & 1230 & 4918 \\
\hline$\left(\mathrm{H}_{2} \mathrm{O}\right)_{35}$ & TZVP & 140 & 910 & 1085 & 4598 \\
\hline$(\mathrm{mU})_{2} \cdot \mathrm{H}_{2} \mathrm{O}$ & $6-31+\mathrm{G}^{* *}$ & 58 & 410 & 489 & 1335 \\
\hline AATT & $6-311+\mathrm{G}^{* *}$ & 98 & 832 & 968 & 1692 \\
\hline Uracil trimer & cc-pVDZ & 62 & 309 & 396 & 1900 \\
\hline Uracil trimer & aug-cc-pVDZ & 62 & 573 & 660 & 2437 \\
\hline Beta carotene & $\mathrm{mTZ}$ & 108 & 884 & 1032 & 4504 \\
\hline GC-dDMP-B & $6-311++\mathrm{G}^{* *}$ & 103 & 892 & 1042 & 3855 \\
\hline Pentacene dimer & aug-cc-pVDZ & 102 & 1118 & 1264 & 4067 \\
\hline
\end{tabular}

15

(H20)_5

$\begin{array}{llrl}\mathrm{O} & 1.53500000 & -5.83100000 & 16.29100000 \\ \mathrm{H} & 1.76700000 & -6.54200000 & 16.93800000 \\ \mathrm{H} & 0.61000000 & -5.55000000 & 16.59499900 \\ \mathrm{O} & 3.65800000 & -9.50900000 & 16.56900000 \\ \mathrm{H} & 4.61200000 & -9.51900000 & 16.31699900 \\ \mathrm{H} & 3.15300000 & -10.12700000 & 16.06699900 \\ \mathrm{O} & 0.07300000 & -9.31000000 & 15.64900000 \\ \mathrm{H} & 0.51400000 & -8.90400000 & 16.41000000\end{array}$




$\begin{array}{rrrr}\mathrm{H} & -0.84600000 & -9.39000000 & 16.00000000 \\ \mathrm{O} & 1.71300000 & -11.03700000 & 18.94000100 \\ \mathrm{H} & 2.66700000 & -10.99600000 & 19.15300000 \\ \mathrm{H} & 1.67200000 & -11.68500000 & 18.20900000 \\ \mathrm{O} & 1.53200000 & -8.06200000 & 17.79999900 \\ \mathrm{H} & 1.92500000 & -8.78400000 & 17.32600000 \\ \mathrm{H} & 1.46800000 & -8.35800000 & 18.71800000\end{array}$

30

(H20)_10

$\begin{array}{lrrr}\mathrm{O} & -1.85800000 & 3.90700000 & -21.11599900 \\ \mathrm{H} & -2.60500000 & 4.00900000 & -21.70800000 \\ \mathrm{H} & -1.35100000 & 3.23300000 & -21.57099900 \\ \mathrm{O} & 1.01100000 & 4.81500000 & -20.33099900 \\ \mathrm{H} & 1.14000000 & 4.15100000 & -19.64699900 \\ \mathrm{H} & 0.02800000 & 4.89500000 & -20.40300000 \\ \mathrm{O} & -1.99500000 & 7.20900000 & -19.09400000 \\ \mathrm{H} & -1.20500000 & 7.38000000 & -19.63400100 \\ \mathrm{H} & -1.78000000 & 6.30200000 & -18.77800000 \\ \mathrm{O} & 0.19700000 & 8.19400000 & -20.60700000 \\ \mathrm{H} & 0.94700000 & 8.14900000 & -19.94199900 \\ \mathrm{H} & 0.61100000 & 8.22000000 & -21.49900100 \\ \mathrm{O} & 2.10900000 & 6.79800000 & -18.70999900 \\ \mathrm{H} & 3.05600000 & 6.96000000 & -18.92799900 \\ \mathrm{H} & 1.88200000 & 5.95900000 & -19.18800000 \\ \mathrm{O} & -2.22000000 & 1.84900000 & -18.02199900 \\ \mathrm{H} & -2.79200000 & 1.71800000 & -17.30999900 \\ \mathrm{H} & -2.74900000 & 1.51300000 & -18.79100000 \\ \mathrm{O} & -1.63100000 & 4.44400000 & -18.44300100 \\ \mathrm{H} & -1.73900000 & 3.52400000 & -18.18300100 \\ \mathrm{H} & -1.81400000 & 4.31100000 & -19.40700000 \\ \mathrm{O} & 1.34700000 & 2.98500000 & -18.42200100 \\ \mathrm{H} & 0.93100000 & 3.20900000 & -17.56500100 \\ \mathrm{H} & 1.08200000 & 2.10600000 & -18.67900100 \\ \mathrm{O} & 0.67500000 & 3.03500000 & -15.72000000 \\ \mathrm{H} & 1.05600000 & 2.13400000 & -15.70600000 \\ \mathrm{H} & -0.23500000 & 2.90600000 & -15.44400000 \\ \mathrm{O} & -0.05500000 & 5.60000000 & -16.55400100 \\ \mathrm{H} & 0.54400000 & 4.94200000 & -16.16700000 \\ \mathrm{H} & -0.58300000 & 5.22200000 & -17.29100000\end{array}$

45

(H2O)_15

$\begin{array}{llll}0 & -8.16500000 & -1.86600000 & 7.11700000\end{array}$ 


\begin{tabular}{|c|c|c|c|}
\hline $\mathrm{H}$ & -7.67000000 & -1.13300000 & 6.69000000 \\
\hline $\mathrm{H}$ & -7.55600000 & -2.21800000 & 7.81100000 \\
\hline 0 & -5.16200000 & -8.03900000 & 4.52900000 \\
\hline $\mathrm{H}$ & -5.61800000 & -8.01300000 & 3.64900000 \\
\hline $\mathrm{H}$ & -4.29500000 & -7.72600000 & 4.32600000 \\
\hline 0 & -8.12200000 & -4.16700000 & 10.24400000 \\
\hline $\mathrm{H}$ & -8.69500000 & -3.35100000 & 10.33000000 \\
\hline $\mathrm{H}$ & -8.81900000 & -4.81000000 & 10.05700000 \\
\hline 0 & -9.78500000 & -5.32300000 & 5.76100000 \\
\hline $\mathrm{H}$ & -10.66300000 & -5.51900000 & 5.48100000 \\
\hline $\mathrm{H}$ & -9.77900000 & -4.31900000 & 5.72500000 \\
\hline 0 & -4.80600000 & -1.82900000 & 7.63400000 \\
\hline $\mathrm{H}$ & -4.39200000 & -2.21300000 & 6.87300000 \\
\hline $\mathrm{H}$ & -5.36900000 & -2.54700000 & 7.92500000 \\
\hline 0 & -9.56800000 & -5.95800000 & 8.73300000 \\
\hline $\mathrm{H}$ & -8.95300000 & -6.69000000 & 8.52200000 \\
\hline $\mathrm{H}$ & -9.66200000 & -5.54500000 & 7.82100000 \\
\hline 0 & -6.23900000 & -3.96600000 & 8.18200000 \\
\hline $\mathrm{H}$ & -6.91600000 & -4.16100000 & 8.84700000 \\
\hline $\mathrm{H}$ & -6.31800000 & -4.73300000 & 7.53500000 \\
\hline 0 & -5.70300000 & -9.34300000 & 6.86000000 \\
\hline $\mathrm{H}$ & -5.52800000 & -8.73600000 & 6.02200000 \\
\hline $\mathrm{H}$ & -4.82300000 & -9.79300000 & 6.94400000 \\
\hline 0 & -7.76400000 & -7.98700000 & 8.44200000 \\
\hline $\mathrm{H}$ & -6.91000000 & -8.20000000 & 7.91400000 \\
\hline $\mathrm{H}$ & -8.02900000 & -8.84500000 & 8.87800000 \\
\hline 0 & -6.61600000 & -4.87600000 & 3.47800000 \\
\hline $\mathrm{H}$ & -6.46300000 & -4.20800000 & 4.12500000 \\
\hline $\mathrm{H}$ & -5.78900000 & -4.98600000 & 3.10600000 \\
\hline 0 & -3.65500000 & -4.49100000 & 6.47300000 \\
\hline $\mathrm{H}$ & -2.99900000 & -3.70900000 & 6.40400000 \\
\hline $\mathrm{H}$ & -3.87000000 & -4.67000000 & 5.59100000 \\
\hline 0 & -10.19600000 & -2.62700000 & 5.47000000 \\
\hline $\mathrm{H}$ & -9.70500000 & -2.45200000 & 4.63200000 \\
\hline $\mathrm{H}$ & -9.57700000 & -2.26200000 & 6.12100000 \\
\hline 0 & -5.75200000 & -2.51500000 & 4.85800000 \\
\hline $\mathrm{H}$ & -5.64600000 & -1.89200000 & 4.11000000 \\
\hline $\mathrm{H}$ & -5.62100000 & -1.95900000 & 5.58900000 \\
\hline 0 & -6.87700000 & -6.01300000 & 6.38200000 \\
\hline $\mathrm{H}$ & -7.81900000 & -6.26200000 & 6.25500000 \\
\hline $\mathrm{H}$ & -6.56800000 & -6.06800000 & 5.45800000 \\
\hline 0 & -4.27900000 & -6.48300000 & 8.11200000 \\
\hline $\mathrm{H}$ & -5.15300000 & -6.64400000 & 7.70000000 \\
\hline $\mathrm{H}$ & -3.94800000 & -5.74800000 & 7.58500000 \\
\hline
\end{tabular}




\begin{tabular}{|c|c|c|c|}
\hline 0 & 9.50300000 & -20.45900000 & -18.92499900 \\
\hline $\mathrm{H}$ & 8.83000000 & -21.01899900 & -18.47400100 \\
\hline $\mathrm{H}$ & 9.90300000 & -19.98200000 & -18.17499900 \\
\hline 0 & 9.70100000 & -18.62000100 & -16.77500000 \\
\hline $\mathrm{H}$ & 9.73800000 & -17.74099900 & -17.06399900 \\
\hline $\mathrm{H}$ & 9.21000000 & -18.55999900 & -15.95400000 \\
\hline 0 & 9.59400000 & -12.66600000 & -18.39699900 \\
\hline $\mathrm{H}$ & 10.40100000 & -13.32300000 & -18.49399900 \\
\hline $\mathrm{H}$ & 10.04500000 & -11.74100000 & -18.39900000 \\
\hline 0 & 5.65500000 & -15.35900000 & -17.38600000 \\
\hline $\mathrm{H}$ & 6.39800000 & -14.74800000 & -17.12200000 \\
\hline $\mathrm{H}$ & 4.92500000 & -14.99800000 & -16.90900000 \\
\hline 0 & 6.05200000 & -16.13299900 & -14.23500000 \\
\hline $\mathrm{H}$ & 5.77900000 & -15.42200000 & -13.61400000 \\
\hline $\mathrm{H}$ & 5.93400000 & -15.66000000 & -15.07100000 \\
\hline 0 & 7.51100000 & -18.67300000 & -21.84900100 \\
\hline $\mathrm{H}$ & 7.79900000 & -17.79500000 & -22.20100000 \\
\hline $\mathrm{H}$ & 7.74700000 & -18.57500100 & -20.89699900 \\
\hline 0 & 10.11400000 & -17.35800000 & -20.23900000 \\
\hline $\mathrm{H}$ & 10.54300000 & -18.12000100 & -20.73100100 \\
\hline $\mathrm{H}$ & 9.90300000 & -16.82000000 & -20.94300100 \\
\hline 0 & 5.84600000 & -15.38400000 & -20.10300100 \\
\hline $\mathrm{H}$ & 6.46600000 & -14.63700000 & -20.13900000 \\
\hline $\mathrm{H}$ & 5.82300000 & -15.57000000 & -19.14500000 \\
\hline 0 & 12.12100000 & -20.16700000 & -16.90700000 \\
\hline $\mathrm{H}$ & 12.87100000 & -19.92900100 & -16.35899900 \\
\hline $\mathrm{H}$ & 11.45600000 & -19.44700100 & -16.67600100 \\
\hline 0 & 11.88300000 & -14.26000000 & -18.95299900 \\
\hline $\mathrm{H}$ & 12.80200000 & -14.48200000 & -18.68899900 \\
\hline $\mathrm{H}$ & 12.05100000 & -13.93100000 & -19.87100000 \\
\hline 0 & 8.04900000 & -14.19200000 & -16.80400100 \\
\hline $\mathrm{H}$ & 8.55200000 & -14.95900000 & -17.01700000 \\
\hline $\mathrm{H}$ & 8.37800000 & -13.55600000 & -17.40099900 \\
\hline 0 & 7.48600000 & -18.36599900 & -15.15400000 \\
\hline $\mathrm{H}$ & 6.76500000 & -18.84199900 & -15.53400000 \\
\hline $\mathrm{H}$ & 7.00800000 & -17.59400000 & -14.86000000 \\
\hline 0 & 8.43900000 & -20.86000100 & -14.39900000 \\
\hline $\mathrm{H}$ & 7.56400000 & -21.06200000 & -14.54000000 \\
\hline $\mathrm{H}$ & 8.48900000 & -19.87900000 & -14.57600000 \\
\hline 0 & 7.10300000 & -21.49399900 & -17.85899900 \\
\hline $\mathrm{H}$ & 6.41700000 & -21.53800000 & -18.52800000 \\
\hline $\mathrm{H}$ & 6.61600000 & -21.13900000 & -17.05699900 \\
\hline 0 & 5.01400000 & -18.18899900 & -18.87599900 \\
\hline $\mathrm{H}$ & 5.94700000 & -18.30100100 & -18.89399900 \\
\hline $\mathrm{H}$ & 4.88200000 & -17.70800000 & -18.03900000 \\
\hline 0 & 12.23300000 & -15.45500000 & -15.11600000 \\
\hline
\end{tabular}




$\begin{array}{rrrr}\mathrm{H} & 12.34300000 & -14.70300000 & -14.53500000 \\ \mathrm{H} & 13.11300000 & -15.91400000 & -14.99800000 \\ \mathrm{O} & 10.58900000 & -17.45400000 & -14.10100000 \\ \mathrm{H} & 11.09200000 & -18.26400000 & -14.21700000 \\ \mathrm{H} & 11.21200000 & -16.74300000 & -14.42000000 \\ \mathrm{O} & 7.71200000 & -18.30999900 & -19.13600000 \\ \mathrm{H} & 8.45100000 & -17.63699900 & -19.25000000 \\ \mathrm{H} & 8.13900000 & -18.89399900 & -18.50400000 \\ \mathrm{O} & 10.26200000 & -15.95200000 & -17.32500100 \\ \mathrm{H} & 10.96600000 & -15.72200000 & -16.69500000 \\ \mathrm{H} & 10.70700000 & -15.96100000 & -18.16200100\end{array}$

60

(H2O) _20

$\begin{array}{lrrr}\mathrm{O} & -15.56900000 & 15.35800000 & 4.19400000 \\ \mathrm{H} & -15.04000000 & 14.66200000 & 3.71600000 \\ \mathrm{H} & -15.40900000 & 15.08000000 & 5.15800000 \\ \mathrm{O} & -16.14200000 & 14.06700000 & 1.76300000 \\ \mathrm{H} & -16.36200000 & 13.14500000 & 1.75900000 \\ \mathrm{H} & -16.34700000 & 14.42300000 & 2.68600000 \\ \mathrm{O} & -16.24300000 & 12.19500000 & 4.75900000 \\ \mathrm{H} & -15.33300000 & 12.18400000 & 4.37300000 \\ \mathrm{H} & -16.16400000 & 12.73600000 & 5.57500000 \\ \mathrm{O} & -20.41400000 & 16.01499900 & 2.73900000 \\ \mathrm{H} & -20.98500100 & 16.56300000 & 2.17900000 \\ \mathrm{H} & -19.63200000 & 15.95100000 & 2.19200000 \\ \mathrm{O} & -14.77900000 & 18.02199900 & 3.71000000 \\ \mathrm{H} & -14.84100000 & 17.02599900 & 3.67500000 \\ \mathrm{H} & -15.23400000 & 18.23600000 & 2.96300000 \\ \mathrm{O} & -12.61800000 & 18.96200000 & 1.56900000 \\ \mathrm{H} & -13.37300000 & 18.36700100 & 1.44400000 \\ \mathrm{H} & -12.97000000 & 19.51600100 & 2.30700000 \\ \mathrm{O} & -18.79200000 & 16.91000000 & 0.38800000 \\ \mathrm{H} & -18.97400100 & 16.37100000 & -0.35300000 \\ \mathrm{H} & -19.59800000 & 17.52800000 & 0.36200000 \\ \mathrm{O} & -17.83400000 & 16.86899900 & 4.36300000 \\ \mathrm{H} & -16.98300000 & 16.56500100 & 4.09100000 \\ \mathrm{H} & -17.95200000 & 16.39399900 & 5.19900000 \\ \mathrm{O} & -16.20599900 & 17.92900100 & 1.24000000 \\ \mathrm{H} & -17.13900000 & 17.63999900 & 1.26500000 \\ \mathrm{H} & -16.17600100 & 18.28300100 & 0.35300000 \\ \mathrm{O} & -16.19400000 & 20.82500100 & 3.30800000 \\ \mathrm{H} & -16.17200100 & 20.76300000 & 2.31900000 \\ \mathrm{H} & -16.33300000 & 21.74600000 & 3.51700000 \\ \mathrm{O} & -18.52700000 & 19.43199900 & 3.24600000 \\ \mathrm{H} & -17.59300000 & 19.74000000 & 3.46100000\end{array}$




$\begin{array}{lrrr}\mathrm{H} & -18.61599900 & 18.61500000 & 3.70300000 \\ \mathrm{O} & -18.36000100 & 15.34800000 & 6.74000000 \\ \mathrm{H} & -19.10500000 & 15.90300000 & 7.20100000 \\ \mathrm{H} & -18.47800100 & 14.49000000 & 7.16600000 \\ \mathrm{O} & -15.94000000 & 19.28700100 & 6.23300000 \\ \mathrm{H} & -15.53400000 & 20.14100100 & 6.31100000 \\ \mathrm{H} & -15.53700000 & 18.83000000 & 5.45800000 \\ \mathrm{O} & -13.82300000 & 12.95900000 & 3.36300000 \\ \mathrm{H} & -13.26700000 & 12.25100000 & 3.58200000 \\ \mathrm{H} & -13.90900000 & 12.94600000 & 2.35800000 \\ \mathrm{O} & -14.10600000 & 15.84100000 & 1.12800000 \\ \mathrm{H} & -14.62900000 & 16.60600100 & 0.79300000 \\ \mathrm{H} & -14.78700000 & 15.17900000 & 1.32100000 \\ \mathrm{O} & -16.08600000 & 14.82100000 & -0.91500000 \\ \mathrm{H} & -16.45999900 & 14.62800000 & -0.04200000 \\ \mathrm{H} & -15.59000000 & 15.65500000 & -0.71900000 \\ \mathrm{O} & -15.18100000 & 13.89100000 & 6.61400000 \\ \mathrm{H} & -14.41300000 & 13.26600000 & 6.75700000 \\ \mathrm{H} & -15.20500000 & 14.54700000 & 7.36000000 \\ \mathrm{O} & -11.90200000 & 14.56900000 & 5.09100000 \\ \mathrm{H} & -12.20400000 & 13.84800000 & 4.57400000 \\ \mathrm{H} & -12.73900000 & 14.97400000 & 5.31700000 \\ \mathrm{O} & -14.86100000 & 17.54800000 & -1.25200000 \\ \mathrm{H} & -15.18100000 & 18.46600000 & -1.23900000 \\ \mathrm{H} & -14.62100000 & 17.43300100 & -2.14200000 \\ \mathrm{O} & -12.48700000 & 18.53000100 & 5.68600000 \\ \mathrm{H} & -12.93100000 & 18.94899900 & 4.87500000 \\ \mathrm{H} & -13.19000000 & 18.05200000 & 6.09800000\end{array}$

72

(H20)_24

$\begin{array}{lrrr}\mathrm{O} & 20.07600000 & -4.87700000 & 6.88200000 \\ \mathrm{H} & 20.66700000 & -5.42600000 & 6.31000000 \\ \mathrm{H} & 19.55600000 & -4.32500000 & 6.28000000 \\ \mathrm{O} & 15.67400000 & -3.43300000 & 8.42300000 \\ \mathrm{H} & 15.09900000 & -3.33300000 & 7.63900000 \\ \mathrm{H} & 16.16700000 & -4.21600000 & 8.12100000 \\ \mathrm{O} & 10.46600000 & -5.37100000 & 6.17000000 \\ \mathrm{H} & 9.53900000 & -5.32600000 & 6.17000000 \\ \mathrm{H} & 10.65300000 & -5.47800000 & 5.24100000 \\ \mathrm{O} & 15.49700000 & -12.53200000 & 5.67900000 \\ \mathrm{H} & 14.73300000 & -12.92200000 & 6.16000000 \\ \mathrm{H} & 15.50700000 & -13.06900000 & 4.93800000 \\ \mathrm{O} & 15.15300000 & -9.75600000 & 5.09900000 \\ \mathrm{H} & 14.43400000 & -9.83300000 & 4.45300000 \\ \mathrm{H} & 15.53000000 & -10.66000000 & 5.10600000\end{array}$




\begin{tabular}{|c|c|c|c|}
\hline 0 & 11.48700000 & -6.66400000 & 2.75000000 \\
\hline $\mathrm{H}$ & 10.92800000 & -6.10100000 & 2.19400000 \\
\hline $\mathrm{H}$ & 11.29900000 & -7.58300000 & 2.48600000 \\
\hline 0 & 16.95999900 & -6.42200000 & 10.79700000 \\
\hline $\mathrm{H}$ & 17.56699900 & -7.18800000 & 10.64500000 \\
\hline $\mathrm{H}$ & 16.72699900 & -6.08500000 & 9.92000000 \\
\hline 0 & 11.41400000 & -4.52800000 & 8.80600000 \\
\hline $\mathrm{H}$ & 10.99000000 & -4.90700000 & 7.99100000 \\
\hline $\mathrm{H}$ & 12.35700000 & -4.60900000 & 8.51500000 \\
\hline 0 & 18.52100000 & -3.50000000 & 4.60400000 \\
\hline $\mathrm{H}$ & 18.95700100 & -3.18200000 & 3.81500000 \\
\hline $\mathrm{H}$ & 18.23600000 & -4.39200000 & 4.37100000 \\
\hline 0 & 15.79700000 & -8.15500000 & 7.30600000 \\
\hline $\mathrm{H}$ & 16.47200000 & -8.71600000 & 7.68300000 \\
\hline $\mathrm{H}$ & 15.59700000 & -8.56900000 & 6.39200000 \\
\hline 0 & 10.00900000 & -7.73200000 & 8.33300000 \\
\hline $\mathrm{H}$ & 10.36500000 & -7.37900000 & 9.16400000 \\
\hline $\mathrm{H}$ & 10.45400000 & -7.17200000 & 7.59500000 \\
\hline 0 & 13.66000000 & -8.27600000 & 9.72300000 \\
\hline $\mathrm{H}$ & 14.10800000 & -8.82300000 & 10.40400000 \\
\hline $\mathrm{H}$ & 14.37200000 & -8.07100000 & 9.05400000 \\
\hline 0 & 13.76400000 & -3.45900000 & 6.04800000 \\
\hline $\mathrm{H}$ & 13.37000000 & -3.85900000 & 6.80400000 \\
\hline $\mathrm{H}$ & 13.43400000 & -4.02900000 & 5.31000000 \\
\hline 0 & 17.03700100 & -5.84600000 & 8.05600000 \\
\hline $\mathrm{H}$ & 17.98600000 & -5.94800000 & 7.84500000 \\
\hline $\mathrm{H}$ & 16.65800100 & -6.64900000 & 7.56100000 \\
\hline 0 & 13.43800000 & -5.83600000 & 7.43600000 \\
\hline $\mathrm{H}$ & 12.96000000 & -6.54200000 & 6.93800000 \\
\hline $\mathrm{H}$ & 14.13200000 & -6.32300000 & 7.88900000 \\
\hline 0 & 16.01899900 & -7.87900000 & 2.62500000 \\
\hline $\mathrm{H}$ & 16.68499900 & -7.17500000 & 2.80200000 \\
\hline $\mathrm{H}$ & 16.07799900 & -8.40300000 & 3.48700000 \\
\hline 0 & 15.65300000 & -4.12100000 & 11.17700000 \\
\hline $\mathrm{H}$ & 15.80400000 & -3.84700000 & 10.30400000 \\
\hline $\mathrm{H}$ & 16.01199900 & -5.04500000 & 11.21600000 \\
\hline 0 & 13.25500000 & -10.35400000 & 3.31700000 \\
\hline $\mathrm{H}$ & 12.66000000 & -10.95800000 & 3.85600000 \\
\hline $\mathrm{H}$ & 12.68300000 & -10.08300000 & 2.57800000 \\
\hline 0 & 13.10400000 & -4.98600000 & 3.80900000 \\
\hline $\mathrm{H}$ & 13.98600000 & -5.27900000 & 3.50700000 \\
\hline $\mathrm{H}$ & 12.47300000 & -5.61100000 & 3.39500000 \\
\hline 0 & 17.67600100 & -9.68300000 & 8.78900000 \\
\hline $\mathrm{H}$ & 17.43100000 & -9.99900000 & 9.68300000 \\
\hline $\mathrm{H}$ & 18.60800000 & -9.38100000 & 8.97300000 \\
\hline 0 & 12.55300000 & -7.87700000 & 6.06500000 \\
\hline $\mathrm{H}$ & 11.78800000 & -8.34000000 & 5.73500000 \\
\hline
\end{tabular}




$\begin{array}{llrl}\mathrm{H} & 13.29200000 & -8.29700000 & 5.62800000 \\ \mathrm{O} & 18.03800000 & -5.77700000 & 2.83000000 \\ \mathrm{H} & 17.91500100 & -5.54900000 & 1.87100000 \\ \mathrm{H} & 18.87200000 & -6.28700000 & 2.72800000 \\ \mathrm{O} & 18.37200000 & -9.73900000 & 3.04800000 \\ \mathrm{H} & 18.22500000 & -10.48400000 & 3.59500000 \\ \mathrm{H} & 17.86100000 & -9.92200000 & 2.25400000 \\ \mathrm{O} & 15.17400000 & -5.42200000 & 1.59500000 \\ \mathrm{H} & 15.46100000 & -6.20800000 & 1.95900000 \\ \mathrm{H} & 15.66000000 & -5.45500000 & 0.76800000\end{array}$

75

(H20)_25

$\begin{array}{llll}\mathrm{O} & -17.49099900 & 13.49800000 & 13.51900000 \\ \mathrm{H} & -18.07500100 & 13.67100000 & 14.31500000 \\ \mathrm{H} & -16.71900000 & 13.18300000 & 13.94000000 \\ \mathrm{O} & -21.73900000 & 16.36300100 & 15.73800000 \\ \mathrm{H} & -21.90300000 & 15.38900000 & 15.77400000 \\ \mathrm{H} & -21.52800000 & 16.43800000 & 14.78700000 \\ \mathrm{O} & -20.71999900 & 20.03700100 & 13.61200000 \\ \mathrm{H} & -21.19599900 & 20.50700000 & 14.33700000 \\ \mathrm{H} & -19.97300000 & 20.58900100 & 13.37900000 \\ \mathrm{O} & -16.55100100 & 16.20999900 & 15.84700000 \\ \mathrm{H} & -16.50400000 & 17.12299900 & 15.80000000 \\ \mathrm{H} & -16.36100000 & 15.90700000 & 14.95300000 \\ \mathrm{O} & -23.70800000 & 19.13800000 & 13.27600000 \\ \mathrm{H} & -23.32900000 & 19.89900000 & 12.94800000 \\ \mathrm{H} & -24.67300000 & 19.32099900 & 13.17500000 \\ \mathrm{O} & -17.59300000 & 11.59700000 & 16.16799900 \\ \mathrm{H} & -18.11100000 & 12.35800000 & 15.91800000 \\ \mathrm{H} & -16.93100000 & 11.97700000 & 16.80800100 \\ \mathrm{O} & -21.72800100 & 16.95299900 & 18.40600000 \\ \mathrm{H} & -22.57600000 & 17.32799900 & 18.70100000 \\ \mathrm{H} & -21.84800000 & 16.87299900 & 17.41400000 \\ \mathrm{O} & -17.40300000 & 14.87800000 & 11.19800000 \\ \mathrm{H} & -17.41700000 & 14.59300000 & 12.10200000 \\ \mathrm{H} & -18.38200000 & 15.05600000 & 11.04300000 \\ \mathrm{O} & -21.37900000 & 12.77000000 & 11.50500000 \\ \mathrm{H} & -21.32600000 & 13.57100000 & 12.02800000 \\ \mathrm{H} & -22.31500100 & 12.84300000 & 11.22600000 \\ \mathrm{O} & -22.47800100 & 13.88000000 & 15.06000000 \\ \mathrm{H} & -22.29200000 & 12.93400000 & 15.04300000 \\ \mathrm{H} & -23.31600000 & 13.90100000 & 15.51100000 \\ \mathrm{O} & -24.11800000 & 16.29999900 & 13.44500000 \\ \mathrm{H} & -23.55600000 & 15.84800000 & 12.85600000 \\ \mathrm{H} & -24.01000000 & 17.24799900 & 13.15100000\end{array}$




\begin{tabular}{|c|c|c|c|}
\hline 0 & -22.58600000 & 11.17500000 & 15.43800000 \\
\hline $\mathrm{H}$ & -22.09300000 & 10.45700000 & 14.94900000 \\
\hline $\mathrm{H}$ & -23.47200000 & 10.83000000 & 15.60100000 \\
\hline 0 & -14.40400000 & 14.20700000 & 14.39200000 \\
\hline $\mathrm{H}$ & -14.36800000 & 15.13200000 & 13.95700000 \\
\hline $\mathrm{H}$ & -14.22200000 & 14.35200000 & 15.29700000 \\
\hline 0 & -22.81900000 & 18.86400000 & 16.04600000 \\
\hline $\mathrm{H}$ & -22.16500100 & 18.19000100 & 15.73900000 \\
\hline $\mathrm{H}$ & -23.09400000 & 19.24799900 & 15.25000000 \\
\hline 0 & -18.23300000 & 17.84300000 & 13.47000000 \\
\hline $\mathrm{H}$ & -17.71699900 & 18.00700000 & 14.26300000 \\
\hline $\mathrm{H}$ & -17.53200000 & 17.97800100 & 12.77200000 \\
\hline 0 & -16.40000000 & 18.81300000 & 15.04700000 \\
\hline $\mathrm{H}$ & -16.09100000 & 19.70700100 & 15.34300000 \\
\hline $\mathrm{H}$ & -15.57800000 & 18.48900000 & 14.51300000 \\
\hline 0 & -20.94500000 & 14.11000000 & 18.06900000 \\
\hline $\mathrm{H}$ & -21.44400000 & 14.30400000 & 18.87299900 \\
\hline $\mathrm{H}$ & -21.67900100 & 13.88600000 & 17.45700100 \\
\hline 0 & -15.19500000 & 16.12900000 & 12.65100000 \\
\hline $\mathrm{H}$ & -15.60500000 & 15.66200000 & 11.86900000 \\
\hline $\mathrm{H}$ & -14.91700000 & 16.96100000 & 12.28900000 \\
\hline 0 & -22.64900000 & 15.89300000 & 10.03700000 \\
\hline $\mathrm{H}$ & -23.28400000 & 15.15500000 & 9.85800000 \\
\hline $\mathrm{H}$ & -23.24200100 & 16.66700000 & 9.91200000 \\
\hline 0 & -19.07600000 & 14.15300000 & 15.80900000 \\
\hline $\mathrm{H}$ & -18.97200000 & 15.06700000 & 15.69400000 \\
\hline $\mathrm{H}$ & -19.82300000 & 14.09100000 & 16.43400000 \\
\hline 0 & -19.07799900 & 11.43800000 & 11.68200000 \\
\hline $\mathrm{H}$ & -18.69100000 & 11.90700000 & 12.42700000 \\
\hline $\mathrm{H}$ & -19.90099900 & 11.96200000 & 11.51300000 \\
\hline 0 & -20.12000100 & 19.31300000 & 10.31300000 \\
\hline $\mathrm{H}$ & -19.19700100 & 19.04400100 & 10.21600000 \\
\hline $\mathrm{H}$ & -20.22900000 & 19.14100100 & 11.25700000 \\
\hline 0 & -16.33799900 & 18.85000000 & 11.23100000 \\
\hline $\mathrm{H}$ & -16.49900100 & 19.04100000 & 10.25200000 \\
\hline $\mathrm{H}$ & -16.16000000 & 19.73300000 & 11.58400000 \\
\hline 0 & -20.91000000 & 17.46600000 & 13.12600000 \\
\hline $\mathrm{H}$ & -19.98500100 & 17.37900000 & 13.17200000 \\
\hline $\mathrm{H}$ & -21.03200000 & 18.40399900 & 13.32500000 \\
\hline 0 & -20.01400000 & 15.52200000 & 11.09500000 \\
\hline $\mathrm{H}$ & -20.85400000 & 15.48300000 & 10.61000000 \\
\hline $\mathrm{H}$ & -20.20000100 & 16.21800000 & 11.81100000 \\
\hline
\end{tabular}

90

(H20)_30

$\begin{array}{llll}0 & -3.08200000 & -8.83200000 & 8.95500000\end{array}$ 


\begin{tabular}{|c|c|c|c|}
\hline $\mathrm{H}$ & -3.26100000 & -9.55100000 & 8.31700000 \\
\hline $\mathrm{H}$ & -3.31800000 & -8.00200000 & 8.51100000 \\
\hline 0 & -3.52500000 & -4.89200000 & 10.28700000 \\
\hline $\mathrm{H}$ & -4.26300000 & -4.67000000 & 10.83200000 \\
\hline $\mathrm{H}$ & -3.94400000 & -5.46500000 & 9.58800000 \\
\hline 0 & 2.02500000 & -5.57000000 & 8.25200000 \\
\hline $\mathrm{H}$ & 2.94100000 & -5.95500000 & 8.23500000 \\
\hline $\mathrm{H}$ & 1.35400000 & -6.22300000 & 8.59900000 \\
\hline 0 & -0.95300000 & -6.83600000 & 7.09500000 \\
\hline $\mathrm{H}$ & -0.56200000 & -7.19200000 & 7.87300000 \\
\hline $\mathrm{H}$ & -1.46500000 & -6.06500000 & 7.34300000 \\
\hline 0 & -0.05200000 & -3.78700000 & 7.94600000 \\
\hline $\mathrm{H}$ & 0.72900000 & -4.31900000 & 7.98600000 \\
\hline $\mathrm{H}$ & -0.67400000 & -4.24800000 & 8.52200000 \\
\hline 0 & 1.56700000 & -3.57000000 & 10.51600000 \\
\hline $\mathrm{H}$ & 1.29100000 & -4.44400000 & 10.24800000 \\
\hline $\mathrm{H}$ & 1.57700000 & -3.04700000 & 9.67700000 \\
\hline 0 & 1.94000000 & -13.31000000 & 6.92500000 \\
\hline $\mathrm{H}$ & 1.91800000 & -12.58200000 & 6.31900000 \\
\hline $\mathrm{H}$ & 1.05200000 & -13.55100000 & 7.00700000 \\
\hline 0 & -0.88900000 & -5.38500000 & 9.93800000 \\
\hline $\mathrm{H}$ & -1.76700000 & -5.30500000 & 10.26100000 \\
\hline $\mathrm{H}$ & -0.42500000 & -5.70800000 & 10.72600000 \\
\hline 0 & 0.97000000 & -5.73500000 & 5.39700000 \\
\hline $\mathrm{H}$ & 1.45000000 & -6.00700000 & 6.19700000 \\
\hline $\mathrm{H}$ & 0.12300000 & -6.17200000 & 5.55400000 \\
\hline 0 & 3.35200000 & -9.00900000 & 13.26100000 \\
\hline $\mathrm{H}$ & 4.03500000 & -9.16700000 & 12.56700000 \\
\hline $\mathrm{H}$ & 3.57400000 & -8.19800000 & 13.71800000 \\
\hline 0 & -4.27900000 & -6.48300000 & 8.11200000 \\
\hline $\mathrm{H}$ & -5.15300000 & -6.64400000 & 7.70000000 \\
\hline $\mathrm{H}$ & -3.94800000 & -5.74800000 & 7.58500000 \\
\hline 0 & -0.68500000 & -11.09800000 & 11.49400000 \\
\hline $\mathrm{H}$ & 0.07200000 & -11.01900000 & 12.09800000 \\
\hline $\mathrm{H}$ & -0.11600000 & -11.22800000 & 10.66000000 \\
\hline 0 & 1.47400000 & -11.46900000 & 4.83000000 \\
\hline $\mathrm{H}$ & 1.03500000 & -11.30100000 & 4.00400000 \\
\hline $\mathrm{H}$ & 2.42000000 & -11.20800000 & 4.71000000 \\
\hline 0 & 3.82300000 & -10.66800000 & 7.29200000 \\
\hline $\mathrm{H}$ & 4.16200000 & -11.13900000 & 8.05000000 \\
\hline $\mathrm{H}$ & 3.16500000 & -10.04000000 & 7.66000000 \\
\hline 0 & 0.06500000 & -5.67400000 & 12.73700000 \\
\hline $\mathrm{H}$ & -0.48000000 & -5.00800000 & 13.12700000 \\
\hline $\mathrm{H}$ & -0.31700000 & -6.54700000 & 13.01600000 \\
\hline 0 & -1.45200000 & -8.47200000 & 10.94200000 \\
\hline $\mathrm{H}$ & -1.28200000 & -9.42900000 & 11.03000000 \\
\hline $\mathrm{H}$ & -2.19200000 & -8.46700000 & 10.31700000 \\
\hline
\end{tabular}




\begin{tabular}{|c|c|c|c|}
\hline 0 & 5.83500000 & -8.74300000 & 11.83200000 \\
\hline $\mathrm{H}$ & 6.45500000 & -7.95100000 & 11.87600000 \\
\hline $\mathrm{H}$ & 5.77600000 & -8.88200000 & 10.82000000 \\
\hline 0 & 1.59600000 & -10.24500000 & 9.44600000 \\
\hline $\mathrm{H}$ & 0.79500000 & -10.31900000 & 8.90800000 \\
\hline $\mathrm{H}$ & 1.69000000 & -11.19200000 & 9.65600000 \\
\hline 0 & 2.65700000 & -6.00200000 & 13.40100000 \\
\hline $\mathrm{H}$ & 1.75500000 & -5.82500000 & 13.12600000 \\
\hline $\mathrm{H}$ & 2.51500000 & -6.66500000 & 14.08300000 \\
\hline 0 & 0.64000000 & -7.84500000 & 9.03900000 \\
\hline $\mathrm{H}$ & 0.00000000 & -7.96000000 & 9.76300000 \\
\hline $\mathrm{H}$ & 1.19000000 & -8.63900000 & 9.14300000 \\
\hline 0 & 4.77800000 & -11.96500000 & 9.96000000 \\
\hline $\mathrm{H}$ & 4.27300000 & -11.94600000 & 10.79400000 \\
\hline $\mathrm{H}$ & 4.53500000 & -12.87400000 & 9.54500000 \\
\hline 0 & 4.05200000 & -5.35300000 & 11.06700000 \\
\hline $\mathrm{H}$ & 4.97300000 & -5.53900000 & 11.25000000 \\
\hline $\mathrm{H}$ & 3.53900000 & -5.53500000 & 11.85800000 \\
\hline 0 & 1.93600000 & -12.74100000 & 10.32000000 \\
\hline $\mathrm{H}$ & 1.62600000 & -13.13900000 & 9.51700000 \\
\hline $\mathrm{H}$ & 2.39600000 & -13.53400000 & 10.63600000 \\
\hline 0 & 1.35600000 & -10.99300000 & 13.53500000 \\
\hline $\mathrm{H}$ & 1.05200000 & -10.42000000 & 14.28700000 \\
\hline $\mathrm{H}$ & 2.16700000 & -10.62600000 & 13.23600000 \\
\hline 0 & -1.60900000 & -7.95900000 & 13.54700000 \\
\hline $\mathrm{H}$ & -0.95800000 & -8.51200000 & 13.92300000 \\
\hline $\mathrm{H}$ & -1.63700000 & -8.16400000 & 12.60300000 \\
\hline 0 & -3.33500000 & -10.86000000 & 6.98100000 \\
\hline $\mathrm{H}$ & -2.68500000 & -11.02800000 & 6.26500000 \\
\hline $\mathrm{H}$ & -3.90300000 & -11.66100000 & 6.97200000 \\
\hline 0 & -1.82300000 & -8.73400000 & 5.19400000 \\
\hline $\mathrm{H}$ & -1.61400000 & -7.99800000 & 5.69600000 \\
\hline $\mathrm{H}$ & -1.52800000 & -9.49900000 & 5.67900000 \\
\hline 0 & 5.81400000 & -9.45300000 & 9.17900000 \\
\hline $\mathrm{H}$ & 5.45400000 & -10.34200000 & 9.28000000 \\
\hline $\mathrm{H}$ & 6.48700000 & -9.59700000 & 8.55700000 \\
\hline 0 & -0.74700000 & -11.30800000 & 8.56100000 \\
\hline $\mathrm{H}$ & -0.74100000 & -12.23900000 & 8.22300000 \\
\hline $\mathrm{H}$ & -1.63400000 & -10.94500000 & 8.50400000 \\
\hline 0 & 4.50800000 & -6.96900000 & 8.70100000 \\
\hline $\mathrm{H}$ & 4.87500000 & -7.81000000 & 8.87500000 \\
\hline $\mathrm{H}$ & 4.25700000 & -6.74800000 & 9.60300000 \\
\hline
\end{tabular}

105

(H20)_35

$\begin{array}{llll}0 & 15.11400000 & -0.89400000 & -12.34600000\end{array}$ 


$\begin{array}{rrrr}\mathrm{H} & 15.59200000 & -0.45700000 & -13.10100000 \\ \mathrm{H} & 15.55300000 & -1.74100000 & -12.23400000 \\ \mathrm{O} & 13.81700000 & -7.74600000 & -12.73300000 \\ \mathrm{H} & 13.49400000 & -8.25700000 & -11.99500000 \\ \mathrm{H} & 14.68500000 & -7.42000000 & -12.37500000 \\ \mathrm{O} & 13.25800000 & -7.73900000 & -8.51100000 \\ \mathrm{H} & 13.62600000 & -6.89200000 & -8.76900000 \\ \mathrm{H} & 12.67600000 & -7.48900000 & -7.85500000 \\ \mathrm{O} & 16.34800000 & -5.11100000 & -6.33100000 \\ \mathrm{H} & 15.99700000 & -4.54000000 & -5.62000000 \\ \mathrm{H} & 17.35899900 & -5.05200000 & -6.25000000 \\ \mathrm{O} & 16.32399900 & -7.81000000 & -11.66700000 \\ \mathrm{H} & 16.55699900 & -6.97300000 & -11.17700000 \\ \mathrm{H} & 16.73000000 & -7.66400000 & -12.48900000 \\ \mathrm{O} & 9.52600000 & -9.84100000 & -9.93200000 \\ \mathrm{H} & 9.18200000 & -9.43100000 & -9.09400000 \\ \mathrm{H} & 9.87500000 & -10.66600000 & -9.59600000 \\ \mathrm{O} & 8.99100000 & -5.70600000 & -13.28900000 \\ \mathrm{H} & 8.38500000 & -5.97300000 & -12.52500000 \\ \mathrm{H} & 8.42900000 & -5.35900000 & -13.98900000 \\ \mathrm{H} & 11.31400000 & -3.11400000 & -9.06900000 \\ \mathrm{H} & 12.93100000 & 0.18400000 & -9.77500000 \\ \mathrm{H} & 13.07800000 & -0.55500000 & -10.36200000 \\ \mathrm{O} & 11.89000000 & -5.84100000 & -13.56000000 \\ \mathrm{H} & 12.50500000 & -6.56300000 & -13.27100000 \\ \mathrm{H} & 11.16000000 & -6.06400000 & -13.07900000 \\ \mathrm{H} & 13.82600000 & 1.00000000 & -10.29200000 \\ \mathrm{H} & 13 & & \end{array}$




\begin{tabular}{|c|c|c|c|}
\hline 0 & 12.58600000 & -1.92600000 & -11.78200000 \\
\hline $\mathrm{H}$ & 13.55000000 & -2.06200000 & -11.80200000 \\
\hline $\mathrm{H}$ & 12.35300000 & -1.48900000 & -12.66800000 \\
\hline 0 & 6.74600000 & -3.36100000 & -12.51200000 \\
\hline $\mathrm{H}$ & 6.67400000 & -2.40900000 & -12.26500000 \\
\hline $\mathrm{H}$ & 6.95800000 & -3.29500000 & -13.46700000 \\
\hline 0 & 16.29000100 & -3.23000000 & -11.55100000 \\
\hline $\mathrm{H}$ & 16.08600000 & -3.78200000 & -12.36100000 \\
\hline $\mathrm{H}$ & 16.27800000 & -3.89600000 & -10.81000000 \\
\hline 0 & 12.38300000 & -0.76400000 & -14.21300000 \\
\hline $\mathrm{H}$ & 11.49700000 & -0.42000000 & -14.04700000 \\
\hline $\mathrm{H}$ & 12.85100000 & -0.14800000 & -14.84100000 \\
\hline 0 & 16.87999900 & -9.30500000 & -9.47600000 \\
\hline $\mathrm{H}$ & 16.62100000 & -8.94200000 & -8.63200000 \\
\hline $\mathrm{H}$ & 16.86800000 & -8.57900000 & -10.17500000 \\
\hline 0 & 12.23000000 & -9.15000000 & -10.71900000 \\
\hline $\mathrm{H}$ & 11.33500000 & -9.38500000 & -10.79100000 \\
\hline $\mathrm{H}$ & 12.26500000 & -8.36600000 & -10.05700000 \\
\hline 0 & 14.19800000 & -10.88900000 & -9.95900000 \\
\hline $\mathrm{H}$ & 13.44900000 & -10.26300000 & -10.13400000 \\
\hline $\mathrm{H}$ & 14.97300000 & -10.31700000 & -10.11400000 \\
\hline 0 & 8.22100000 & -3.69400000 & -10.03600000 \\
\hline $\mathrm{H}$ & 7.73400000 & -3.46800000 & -10.87100000 \\
\hline $\mathrm{H}$ & 9.18400000 & -3.59000000 & -10.31700000 \\
\hline 0 & 13.73400000 & -5.37500000 & -15.62900000 \\
\hline $\mathrm{H}$ & 13.59300000 & -5.92200000 & -16.39399900 \\
\hline $\mathrm{H}$ & 13.08500000 & -5.65100000 & -14.97300000 \\
\hline 0 & 14.54900000 & -4.88200000 & -8.37100000 \\
\hline $\mathrm{H}$ & 15.09800000 & -4.90900000 & -7.59900000 \\
\hline $\mathrm{H}$ & 13.94300000 & -4.17000000 & -8.15100000 \\
\hline 0 & 13.44000000 & 1.61100000 & -12.22200000 \\
\hline $\mathrm{H}$ & 12.93500000 & 1.54200000 & -13.06000000 \\
\hline $\mathrm{H}$ & 13.95500000 & 0.81700000 & -12.30400000 \\
\hline 0 & 13.80900000 & -9.89200000 & -14.54700000 \\
\hline $\mathrm{H}$ & 13.87200000 & -9.03200000 & -14.06200000 \\
\hline $\mathrm{H}$ & 14.62300000 & -10.35500000 & -14.23100000 \\
\hline 0 & 17.91200100 & -2.87100000 & -14.55700000 \\
\hline $\mathrm{H}$ & 18.05600000 & -3.56900000 & -15.17900000 \\
\hline $\mathrm{H}$ & 17.00799900 & -3.12700000 & -14.22100000 \\
\hline 0 & 12.99500000 & -2.77800000 & -7.81600000 \\
\hline $\mathrm{H}$ & 12.44800000 & -2.71500000 & -6.97200000 \\
\hline $\mathrm{H}$ & 13.48900000 & -1.88700000 & -7.76900000 \\
\hline 0 & 15.66800000 & -4.46100000 & -13.85400000 \\
\hline $\mathrm{H}$ & 16.04900000 & -5.37300000 & -13.87400000 \\
\hline $\mathrm{H}$ & 14.87700000 & -4.51700000 & -14.39200000 \\
\hline 0 & 15.83000000 & -3.16700000 & -17.07600000 \\
\hline $\mathrm{H}$ & 15.14000000 & -3.70200000 & -16.70900000 \\
\hline
\end{tabular}




$\begin{array}{llll}\mathrm{H} & 15.32200000 & -2.61600000 & -17.69300100 \\ \mathrm{O} & 16.88400100 & -6.84000000 & -14.18500000 \\ \mathrm{H} & 17.59800000 & -6.37500000 & -14.62400000 \\ \mathrm{H} & 16.82300000 & -7.63500000 & -14.74100000 \\ \mathrm{O} & 10.52200000 & -6.42400000 & -9.52700000 \\ \mathrm{H} & 10.72300000 & -6.38900000 & -8.57700000 \\ \mathrm{H} & 10.99400000 & -5.61100000 & -9.81900000 \\ \mathrm{O} & 16.83499900 & -5.77100000 & -9.96500000 \\ \mathrm{H} & 17.59400000 & -5.48900000 & -9.41200000 \\ \mathrm{H} & 16.05699900 & -5.55700000 & -9.36100000\end{array}$

39

(mU)_2 . H2O

\begin{tabular}{|c|c|c|c|}
\hline $\mathrm{C}$ & 1.545064 & -1.804675 & 1.545512 \\
\hline $\mathrm{N}$ & 0.285229 & -1.677479 & 0.814467 \\
\hline $\mathrm{C}$ & 0.166397 & -2.272594 & -0.454951 \\
\hline C & -1.158084 & -2.205915 & -1.027086 \\
\hline $\mathrm{C}$ & -2.123943 & -1.485110 & -0.421915 \\
\hline $\mathrm{N}$ & -1.915212 & -0.838218 & 0.765459 \\
\hline C & -0.711456 & -0.945786 & 1.443811 \\
\hline 0 & -0.534470 & -0.411004 & 2.527996 \\
\hline C & -2.963688 & -0.035285 & 1.390765 \\
\hline $\mathrm{H}$ & -2.554141 & 0.934756 & 1.673037 \\
\hline $\mathrm{H}$ & -3.754361 & 0.115182 & 0.656665 \\
\hline $\mathrm{H}$ & -3.348163 & -0.532963 & 2.284131 \\
\hline $\mathrm{H}$ & -3.103137 & -1.340172 & -0.861695 \\
\hline $\mathrm{H}$ & -1.323978 & -2.684906 & -1.981367 \\
\hline 0 & 1.140501 & -2.781078 & -1.001905 \\
\hline $\mathrm{H}$ & 1.993313 & -0.823547 & 1.711752 \\
\hline $\mathrm{H}$ & 1.361558 & -2.269153 & 2.515745 \\
\hline $\mathrm{H}$ & 2.202198 & -2.425695 & 0.941117 \\
\hline $\mathrm{N}$ & 2.116658 & 0.486447 & -0.992980 \\
\hline C & 1.138568 & 0.344926 & -1.934601 \\
\hline $\mathrm{H}$ & 1.385874 & -0.312748 & -2.759747 \\
\hline C & -0.064934 & 0.942510 & -1.826581 \\
\hline $\mathrm{H}$ & -0.842390 & 0.791306 & -2.561735 \\
\hline $\mathrm{C}$ & -0.368309 & 1.729061 & -0.655415 \\
\hline 0 & -1.466447 & 2.252072 & -0.438769 \\
\hline $\mathrm{N}$ & 0.670508 & 1.858510 & 0.264464 \\
\hline $\mathrm{C}$ & 0.451963 & 2.605626 & 1.505261 \\
\hline $\mathrm{H}$ & 1.218312 & 3.374252 & 1.608281 \\
\hline $\mathrm{H}$ & -0.538173 & 3.050400 & 1.453806 \\
\hline $\mathrm{H}$ & 0.510460 & 1.916115 & 2.349498 \\
\hline $\mathrm{C}$ & 1.929937 & 1.284546 & 0.127945 \\
\hline 0 & 2.806299 & 1.456191 & 0.958016 \\
\hline $\mathrm{C}$ & 3.395448 & -0.205179 & -1.147009 \\
\hline
\end{tabular}




$\begin{array}{rrrr}\mathrm{H} & 4.063549 & 0.371938 & -1.791451 \\ \mathrm{H} & 3.849480 & -0.298544 & -0.163000 \\ \mathrm{H} & 3.207981 & -1.196199 & -1.563228 \\ \mathrm{O} & -3.551228 & 0.952796 & -1.702837 \\ \mathrm{H} & -4.133353 & 1.534797 & -2.195201 \\ \mathrm{H} & -2.874319 & 1.525783 & -1.290891\end{array}$

60

AATT

$\mathrm{N}$

4.933372

$-0.652401$

1.567963

C

4.782400

0.482761

2. 335118

0.774553

2. 612804

2.808187

$-0.230005$

1. 978375

1. 430371

$-0.498997$

1.888625

0.242012

2.472008

0.484454

$-1.577325$

1. 168217

1.996652

$-2.327480$

0.583704

$-2.181833$

0.600479

$-1.105872$

1. 324195

3.660963

0.062237

$-2.370046$

1.044161

$-1.595124$

5.222661

1.743856

$-0.892815$

1. 191641

$-1.234691$

1. 473710

$-0.820053$

2.412334

0.063341

0.730580

$-1.347517$

$-0.233683$

$-2.222372$

$-0.597666$

$-2.682467$

0.159535

$-2.145551$

1. 175240

$-1.668947$

0.852210

$-1.845154$

0.134394

$-2.748531$

1. 401121

$-0.916872$

2. 205332

0.157303

2. 644961

0.899530

2.470201

0.309209

3. 291113

1.475440

1. 955432

$-0.607897$

$-2.936903$

$-1.004080$

$-2.710900$

$-0.630457$

$-3.167912$

$-1.243704$

$-1.922963$

0.479694

$-1.336214$

1. 225506

$-0.645842$

2. 195441

$-1.598884$

0.766076

$-0.995328$

1.511021 


$\begin{array}{rrrr}\mathrm{C} & -4.143924 & -2.378097 & -0.316323 \\ \mathrm{H} & 5.634063 & 1.064617 & 2.657101 \\ \mathrm{H} & 0.746500 & 1.082317 & 2.959601 \\ \mathrm{H} & -0.501938 & -0.002634 & 2.391471 \\ \mathrm{H} & 1.608242 & -3.155126 & -0.004534 \\ \mathrm{H} & 6.291440 & 1.207255 & -1.586397 \\ \mathrm{H} & 2.216641 & 2.881413 & 0.561742 \\ \mathrm{H} & 0.519215 & 2.485870 & 0.406134 \\ \mathrm{H} & 0.269720 & -0.796087 & -2.601331 \\ \mathrm{H} & -0.930607 & 1.148159 & -1.047409 \\ \mathrm{H} & -5.240856 & 3.424662 & 1.455065 \\ \mathrm{H} & -3.680063 & 4.275568 & 1.470584 \\ \mathrm{H} & -3.878536 & 2.804296 & 2.415249 \\ \mathrm{H} & -5.602410 & 2.125849 & -0.555843 \\ \mathrm{H} & -0.639080 & -1.781127 & 0.770889 \\ \mathrm{H} & -6.090057 & -1.247239 & 1.035981 \\ \mathrm{H} & -5.040838 & 0.094030 & 1.551165 \\ \mathrm{H} & -5.161876 & -1.348358 & 2.546381 \\ \mathrm{H} & -5.126930 & -2.611972 & -0.712652 \\ \mathrm{H} & 5.785419 & -1.049962 & 1.208988 \\ \mathrm{H} & 5.101053 & -0.601273 & -2.978111 \\ \mathrm{H} & -4.769876 & 0.803247 & -2.342853 \\ \mathrm{H} & -3.229023 & -3.459136 & -1.855586 \\ & & & \end{array}$

36

Uracil Trimer

$\begin{array}{lrrr}\mathrm{C} & -0.846270333 & 0.026602486 & -0.891883692 \\ \mathrm{C} & 0.262735419 & -0.092177277 & 1.308696879 \\ \mathrm{C} & 1.525282750 & 0.130642666 & 0.556485510 \\ \mathrm{C} & 1.557224140 & 0.000127755 & -0.745764043 \\ \mathrm{~N} & -0.849139249 & 0.015953686 & 0.460923282 \\ \mathrm{~N} & 0.390195164 & -0.133872122 & -1.479060629 \\ \mathrm{O} & -1.860209301 & 0.067657314 & -1.644844731 \\ \mathrm{O} & 0.170179461 & -0.023218747 & 2.491068426 \\ \mathrm{H} & -1.825285936 & 0.070789717 & 0.708302566 \\ \mathrm{H} & 2.419500052 & 0.196390960 & 1.089227731 \\ \mathrm{H} & 2.437832898 & 0.209195489 & -1.356857246 \\ \mathrm{H} & 0.411851650 & -0.319521620 & -2.433716402 \\ \mathrm{C} & -0.846270333 & 8.026602486 & -0.891883692 \\ \mathrm{C} & 0.262735419 & 8.092177277 & 1.308696879 \\ \mathrm{C} & 1.525282750 & 8.130642666 & 0.556485510 \\ \mathrm{C} & 1.557224140 & 8.000127755 & -0.745764043 \\ \mathrm{~N} & -0.849139249 & 8.015953686 & 0.460923282 \\ \mathrm{~N} & 0.390195164 & 8.133872122 & -1.479060629 \\ \mathrm{O} & -1.860209301 & 8.067657314 & -1.644844731 \\ \mathrm{O} & 0.170179461 & 8.023218747 & 2.491068426\end{array}$




$\begin{array}{rrrr}\mathrm{H} & -1.825285936 & 8.070789717 & 0.708302566 \\ \mathrm{H} & 2.419500052 & 8.196390960 & 1.089227731 \\ \mathrm{H} & 2.437832898 & 8.209195489 & -1.356857246 \\ \mathrm{H} & 0.411851650 & 8.319521620 & -2.433716402 \\ \mathrm{C} & -0.846270333 & 4.026602486 & -0.891883692 \\ \mathrm{C} & 0.262735419 & 4.092177277 & 1.308696879 \\ \mathrm{C} & 1.525282750 & 4.130642666 & 0.556485510 \\ \mathrm{C} & 1.557224140 & 4.000127755 & -0.745764043 \\ \mathrm{~N} & -0.849139249 & 4.015953686 & 0.460923282 \\ \mathrm{~N} & 0.390195164 & 4.133872122 & -1.479060629 \\ \mathrm{O} & -1.860209301 & 4.067657314 & -1.644844731 \\ \mathrm{O} & 0.170179461 & 4.023218747 & 2.491068426 \\ \mathrm{H} & -1.825285936 & 4.070789717 & 0.708302566 \\ \mathrm{H} & 2.419500052 & 4.196390960 & 1.089227731 \\ \mathrm{H} & 2.437832898 & 4.209195489 & -1.356857246 \\ \mathrm{H} & 0.411851650 & 4.319521620 & -2.433716402\end{array}$

96

Beta Carotene

$\begin{array}{lrrr}\text { C } & -12.8901 & -0.8145 & -0.5076 \\ \text { C } & 12.8997 & 0.4410 & 0.6411 \\ \text { C } & -14.2559 & -0.0801 & -0.4187 \\ \text { C } & 13.9687 & -0.5754 & 1.1283 \\ \text { C } & -14.1325 & 1.4021 & -0.7232 \\ \text { C } & 14.2584 & -1.6458 & 0.0914 \\ \text { C } & -11.7656 & -0.0110 & 0.1873 \\ \text { C } & 11.7051 & -0.2704 & -0.0369 \\ \text { C } & -13.1937 & 2.0628 & 0.2749 \\ \text { C } & 12.9914 & -2.4292 & -0.2171 \\ \text { C } & -11.9254 & 1.2838 & 0.5600 \\ \text { C } & 11.7507 & -1.5779 & -0.3966 \\ \text { C } & -12.5294 & -1.0270 & -1.9959 \\ \text { C } & -13.1074 & -2.1999 & 0.1487 \\ \text { C } & 13.5556 & 1.4186 & -0.3613 \\ \text { C } & 12.4673 & 1.2391 & 1.8955 \\ \text { C } & -10.5162 & -0.7258 & 0.3915 \\ \text { C } & 10.5386 & 0.5604 & -0.2876 \\ \text { C } & -10.9033 & 2.1251 & 1.2890 \\ \text { C } & 10.6162 & -2.3640 & -1.0125 \\ \text { C } & -9.3101 & -0.1674 & 0.2000 \\ \text { C } & 9.2794 & 0.1212 & -0.1333 \\ \text { C } & -8.0143 & -0.8203 & 0.3882 \\ \text { C } & 8.0576 & 0.8903 & -0.3702 \\ \text { C } & -8.0214 & -2.2597 & 0.8498 \\ \text { C } & 8.2067 & 2.3079 & -0.8682 \\ \text { C } & -6.8238 & -0.2163 & 0.1826\end{array}$




\begin{tabular}{|c|c|c|c|}
\hline C & 6.8152 & 0.3903 & -0.1964 \\
\hline $\mathrm{C}$ & -5.4906 & -0.7696 & 0.3389 \\
\hline C & 5.5378 & 1.0456 & -0.3959 \\
\hline C & -4.3789 & -0.0528 & 0.0989 \\
\hline C & 4.3476 & 0.4561 & -0.1936 \\
\hline C & -2.9988 & -0.5222 & 0.2249 \\
\hline C & 3.0244 & 1.0661 & -0.3595 \\
\hline C & -2.7772 & -1.9381 & 0.6986 \\
\hline C & 2.9783 & 2.4996 & -0.8248 \\
\hline C & -1.9244 & 0.2540 & -0.0330 \\
\hline C & 1.9379 & 0.3050 & -0.1059 \\
\hline C & -0.5200 & -0.0913 & 0.0540 \\
\hline $\mathrm{C}$ & 0.5427 & 0.6895 & -0.2053 \\
\hline $\mathrm{H}$ & -14.9809 & -0.5336 & -1.1065 \\
\hline $\mathrm{H}$ & -14.6780 & -0.1942 & 0.5896 \\
\hline $\mathrm{H}$ & 14.9007 & -0.0578 & 1.3881 \\
\hline $\mathrm{H}$ & 13.6261 & -1.0679 & 2.0491 \\
\hline $\mathrm{H}$ & -15.1180 & 1.8792 & -0.6708 \\
\hline $\mathrm{H}$ & -13.7657 & 1.5561 & -1.7445 \\
\hline $\mathrm{H}$ & 15.0309 & -2.3289 & 0.4631 \\
\hline $\mathrm{H}$ & 14.6555 & -1.1947 & -0.8253 \\
\hline $\mathrm{H}$ & -12.9377 & 3.0589 & -0.1072 \\
\hline $\mathrm{H}$ & -13.7245 & 2.2123 & 1.2241 \\
\hline $\mathrm{H}$ & 13.1799 & -3.0184 & -1.1229 \\
\hline $\mathrm{H}$ & 12.7999 & -3.1441 & 0.5935 \\
\hline $\mathrm{H}$ & -13.3418 & -1.5315 & -2.5315 \\
\hline $\mathrm{H}$ & -11.6340 & -1.6498 & -2.1064 \\
\hline $\mathrm{H}$ & -12.3301 & -0.0788 & -2.5070 \\
\hline $\mathrm{H}$ & -12.2784 & -2.8883 & -0.0477 \\
\hline $\mathrm{H}$ & -14.0093 & -2.6823 & -0.2474 \\
\hline $\mathrm{H}$ & -13.2289 & -2.1122 & 1.2346 \\
\hline $\mathrm{H}$ & 12.8973 & 2.2601 & -0.6035 \\
\hline $\mathrm{H}$ & 13.8132 & 0.9231 & -1.3038 \\
\hline $\mathrm{H}$ & 14.4766 & 1.8465 & 0.0515 \\
\hline $\mathrm{H}$ & 13.3367 & 1.6674 & 2.4081 \\
\hline $\mathrm{H}$ & 11.9395 & 0.5977 & 2.6110 \\
\hline $\mathrm{H}$ & 11.8023 & 2.0723 & 1.6424 \\
\hline $\mathrm{H}$ & -10.5940 & -1.7437 & 0.7546 \\
\hline $\mathrm{H}$ & 10.7350 & 1.5667 & -0.6416 \\
\hline $\mathrm{H}$ & -11.4085 & 2.7969 & 1.9939 \\
\hline $\mathrm{H}$ & $-10 \cdot 3430$ & 2.7487 & 0.5854 \\
\hline $\mathrm{H}$ & -10.2131 & 1.5415 & 1.9032 \\
\hline $\mathrm{H}$ & 10.0172 & -2.8518 & -0.2374 \\
\hline $\mathrm{H}$ & 11.0083 & -3.1587 & -1.6589 \\
\hline $\mathrm{H}$ & 9.9808 & -1.7688 & -1.6729 \\
\hline $\mathrm{H}$ & -9.2338 & 0.8426 & -0.1967 \\
\hline $\mathrm{H}$ & 9.0889 & -0.8649 & 0.2815 \\
\hline
\end{tabular}




$\begin{array}{rrrr}\mathrm{H} & -7.0523 & -2.7392 & 0.9778 \\ \mathrm{H} & -8.5673 & -2.8803 & 0.1289 \\ \mathrm{H} & -8.5251 & -2.3381 & 1.8208 \\ \mathrm{H} & 8.7409 & 2.9103 & -0.1238 \\ \mathrm{H} & 7.2876 & 2.8471 & -1.0927 \\ \mathrm{H} & 8.7877 & 2.3189 & -1.7983 \\ \mathrm{H} & -6.8440 & 0.8237 & -0.1458 \\ \mathrm{H} & 6.7362 & -0.6420 & 0.1473 \\ \mathrm{H} & -5.3867 & -1.7974 & 0.6561 \\ \mathrm{H} & 5.5437 & 2.0792 & -0.7140 \\ \mathrm{H} & -4.4832 & 0.9825 & -0.2248 \\ \mathrm{H} & 4.3471 & -0.5839 & 0.1336 \\ \mathrm{H} & -3.2750 & -2.0922 & 1.6638 \\ \mathrm{H} & -3.2048 & -2.6440 & -0.0234 \\ \mathrm{H} & -1.7457 & -2.2497 & 0.8542 \\ \mathrm{H} & 3.4831 & 3.1529 & -0.1041 \\ \mathrm{H} & 1.9677 & 2.8941 & -0.9455 \\ \mathrm{H} & 3.4696 & 2.6034 & -1.7990 \\ \mathrm{H} & -2.1061 & 1.2785 & -0.3592 \\ \mathrm{H} & 2.0859 & -0.7259 & 0.2177 \\ \mathrm{H} & -0.2764 & -1.1040 & 0.3568 \\ \mathrm{H} & 0.3341 & 1.7091 & -0.5157\end{array}$

63

GC-dDMP-B

$\begin{array}{lrrr}\mathrm{O} & 4.82377234 & 1.68139077 & 0.90019282 \\ \mathrm{C} & 4.71063317 & 2.36148719 & -0.35027019 \\ \mathrm{C} & 3.28496053 & 2.23497601 & -0.84829082 \\ \mathrm{O} & 3.03936937 & 0.87266207 & -1.23459162 \\ \mathrm{C} & 2.21789971 & 2.58959387 & 0.19597881 \\ \mathrm{O} & 1.13566601 & 3.19850430 & -0.54089210 \\ \mathrm{C} & 1.85545879 & 1.22659558 & 0.76216654 \\ \mathrm{C} & 1.93774007 & 0.35834729 & -0.48545094 \\ \mathrm{~N} & 2.15226975 & -1.03994068 & -0.21498873 \\ \mathrm{C} & 3.05095832 & -1.61847920 & 0.65494246 \\ \mathrm{~N} & 2.88073851 & -2.93108821 & 0.78199367 \\ \mathrm{C} & 1.82226128 & -3.21293764 & -0.05632504 \\ \mathrm{C} & 1.08303869 & -4.43786125 & -0.23647337 \\ \mathrm{O} & 1.25378958 & -5.53811379 & 0.26979174 \\ \mathrm{~N} & 0.00951905 & -4.21586641 & -1.14280978 \\ \mathrm{C} & -0.41837485 & -2.99930254 & -1.62082067 \\ \mathrm{~N} & -1.57523945 & -2.99648906 & -2.37044885 \\ \mathrm{~N} & 0.24720839 & -1.87809539 & -1.44822601 \\ \mathrm{C} & 1.34634514 & -2.05546407 & -0.67325198 \\ \mathrm{P} & -0.33544359 & 3.38842225 & 0.13226405 \\ \mathrm{O} & -1.01647708 & 4.57532796 & -0.51585028\end{array}$




\begin{tabular}{|c|c|c|c|}
\hline 0 & -0.25781022 & 3.43810027 & 1.64589973 \\
\hline 0 & -1.09978236 & 1.99968689 & -0.26693348 \\
\hline $\mathrm{C}$ & -1.52900436 & 1.81158956 & -1.62577139 \\
\hline $\mathrm{C}$ & -2.80617619 & 0.99472029 & -1.61209213 \\
\hline 0 & -2.52509864 & -0.35409021 & -1.22868567 \\
\hline $\mathrm{C}$ & -3.86820903 & 1.50693629 & -0.62698399 \\
\hline 0 & -5.19048948 & 1.20852723 & -1.07625370 \\
\hline $\mathrm{C}$ & -3.62869406 & 0.65394194 & 0.61030791 \\
\hline C & -3.18292903 & -0.67972698 & 0.00899223 \\
\hline $\mathrm{N}$ & -2.26753527 & -1.45633307 & 0.82609276 \\
\hline $\mathrm{C}$ & -2.38938460 & -2.88051764 & 0.80586682 \\
\hline 0 & -3.28001569 & -3.40289680 & 0.13767935 \\
\hline $\mathrm{N}$ & -1.48093669 & -3.60103385 & 1.53895342 \\
\hline $\mathrm{C}$ & -0.44005736 & -3.00408173 & 2.09439652 \\
\hline $\mathrm{N}$ & 0.43118699 & -3.78087697 & 2.80341956 \\
\hline C & -0.21156272 & -1.59024030 & 2.00657679 \\
\hline $\mathrm{C}$ & -1.15315192 & -0.85978836 & 1.34931661 \\
\hline $\mathrm{H}$ & 5.76080092 & 1.55963387 & 1.07915378 \\
\hline $\mathrm{H}$ & 4.95595412 & 3.42678646 & -0.23670810 \\
\hline $\mathrm{H}$ & 5.37207701 & 1.91647049 & -1.10247267 \\
\hline $\mathrm{H}$ & 2.63015316 & 0.93639807 & 1.47549241 \\
\hline $\mathrm{H}$ & 0.88106002 & 1.20557473 & 1. 24665081 \\
\hline $\mathrm{H}$ & -2.84619396 & 1.10230592 & 1.22280276 \\
\hline $\mathrm{H}$ & -4.54482588 & 0.54272735 & 1.19476806 \\
\hline $\mathrm{H}$ & -4.02956685 & -1.33380396 & -0.20050941 \\
\hline $\mathrm{H}$ & -3.74990991 & 2.58056040 & -0.43512314 \\
\hline $\mathrm{H}$ & -3.21142960 & 0.98197257 & -2.63470236 \\
\hline $\mathrm{H}$ & -1.71678020 & 2.78483458 & -2.09508631 \\
\hline $\mathrm{H}$ & -0.75588866 & 1.27026777 & -2.18181582 \\
\hline $\mathrm{H}$ & 1.01728262 & 0.40626426 & -1.07572102 \\
\hline $\mathrm{H}$ & 2.57386242 & 3.28790013 & 0.95805605 \\
\hline $\mathrm{H}$ & 3.15475236 & 2.88052127 & -1.72457105 \\
\hline $\mathrm{H}$ & -0.61346532 & -5.01367962 & -1.23490456 \\
\hline $\mathrm{H}$ & -2.28213949 & -3.63386045 & -2.01335723 \\
\hline $\mathrm{H}$ & -1.95192405 & -2.05104973 & -2.39741587 \\
\hline $\mathrm{H}$ & 3.82784247 & -1.03623481 & 1.13216309 \\
\hline $\mathrm{H}$ & 1.39192646 & -3.45761088 & 2.82280403 \\
\hline $\mathrm{H}$ & 0.35010172 & -4.77028926 & 2.59230156 \\
\hline $\mathrm{H}$ & 0.66531013 & -1.12039147 & 2.43705584 \\
\hline $\mathrm{H}$ & -1.06457375 & 0.20789611 & 1.18623221 \\
\hline $\mathrm{Na}$ & -1.30760258 & 5.48305670 & 1.57691474 \\
\hline $\mathrm{H}$ & -5.31495133 & 1.64129795 & -1.92789922 \\
\hline
\end{tabular}

72

Pentacene Dimer

$\begin{array}{llll}\text { C } & -0.72537787 & 0.32890493 & -1.16524187\end{array}$ 


\begin{tabular}{|c|c|c|c|}
\hline $\mathrm{C}$ & -0.51023340 & 0.38305041 & 1.25899737 \\
\hline $\mathrm{C}$ & 0.51023340 & -0.38305041 & -1.25899737 \\
\hline $\mathrm{C}$ & 0.72537787 & -0.32890493 & 1.16524187 \\
\hline C & -1.20766964 & 0.69585546 & 0.09163535 \\
\hline C & 1.20766964 & -0.69585546 & -0.09163535 \\
\hline C & 0.29160582 & -0.43556160 & -3.68807885 \\
\hline $\mathrm{C}$ & 0.93793954 & -0.27289858 & 3.59478361 \\
\hline C & -0.93793954 & 0.27289858 & -3.59478361 \\
\hline $\mathrm{C}$ & -0.29160582 & 0.43556160 & 3.68807885 \\
\hline C & 0.99088295 & -0.75068114 & -2.53803636 \\
\hline C & 1.42510113 & -0.64140131 & 2.35471673 \\
\hline C & -1.42510113 & 0.64140131 & -2.35471673 \\
\hline $\mathrm{C}$ & -0.99088295 & 0.75068114 & 2.53803636 \\
\hline C & 0.07391953 & -0.48762907 & -6.10372002 \\
\hline C & 1.14906028 & -0.21704806 & 6.01092297 \\
\hline $\mathrm{C}$ & -1.14906028 & 0.21704806 & -6.01092297 \\
\hline C & -0.07391953 & 0.48762907 & 6.10372002 \\
\hline $\mathrm{C}$ & 0.77459321 & -0.80620762 & -4.99015421 \\
\hline $\mathrm{C}$ & 1.64403085 & -0.58739597 & 4.80663426 \\
\hline $\mathrm{C}$ & -1.64403085 & 0.58739597 & -4.80663426 \\
\hline $\mathrm{C}$ & -0.77459321 & 0.80620762 & 4.99015421 \\
\hline $\mathrm{H}$ & -2.13551484 & 1.23047695 & 0.16203823 \\
\hline $\mathrm{H}$ & 2.13551484 & -1.23047695 & -0.16203823 \\
\hline $\mathrm{H}$ & -2.35301943 & 1.17587141 & -2.28695991 \\
\hline $\mathrm{H}$ & -1.91833375 & 1.28526889 & 2.61106092 \\
\hline $\mathrm{H}$ & 1.91833375 & -1.28526889 & -2.61106092 \\
\hline $\mathrm{H}$ & 2.35301943 & -1.17587141 & 2.28695991 \\
\hline $\mathrm{H}$ & -1.68534529 & 0.45758427 & -6.90843347 \\
\hline $\mathrm{H}$ & -0.44479268 & 0.76979454 & 7.07006372 \\
\hline $\mathrm{H}$ & 0.44479268 & -0.76979454 & -7.07006372 \\
\hline $\mathrm{H}$ & 1.68534529 & -0.45758427 & 6.90843347 \\
\hline $\mathrm{H}$ & -2.57159898 & 1.12174357 & -4.73781787 \\
\hline $\mathrm{H}$ & -1.70188534 & 1.34062468 & 5.06208058 \\
\hline $\mathrm{H}$ & 1.70188534 & -1.34062468 & -5.06208058 \\
\hline $\mathrm{H}$ & 2.57159898 & -1.12174357 & 4.73781787 \\
\hline $\mathrm{C}$ & 2.24846286 & 3.41094746 & -1.13506502 \\
\hline $\mathrm{C}$ & 2.50212283 & 3.48153794 & 1.28502348 \\
\hline $\mathrm{C}$ & 3.48339217 & 4.11441606 & -1.28502248 \\
\hline C & 3.73705214 & 4.18500654 & 1.13506602 \\
\hline C & 1.78575440 & 3.11041636 & 0.14656688 \\
\hline C & 4.19976060 & 4.48553764 & -0.14656588 \\
\hline C & 3.22617016 & 4.04195114 & -3.70980658 \\
\hline $\mathrm{C}$ & 3.98821158 & 4.25401797 & 3.56058630 \\
\hline $\mathrm{C}$ & 1.99730342 & 3.34193603 & -3.56058530 \\
\hline $\mathrm{C}$ & 2.75934484 & 3.55400286 & 3.70980758 \\
\hline $\mathrm{C}$ & 3.94410651 & 4.41448611 & -2.58879292 \\
\hline $\mathrm{C}$ & 4.45605913 & 4.55695627 & 2.29558290 \\
\hline
\end{tabular}




\begin{tabular}{lrrr} 
C & 1.52945587 & 3.03899773 & -2.29558190 \\
C & 2.04140849 & 3.18146789 & 2.58879392 \\
C & 2.97010282 & 3.96973480 & -6.12116200 \\
C & 4.23771700 & 4.32249635 & 5.97273853 \\
C & 1.74779800 & 3.27345765 & -5.97273753 \\
C & 3.01541218 & 3.62621920 & 6.12116300 \\
C & 3.68885920 & 4.34383827 & -5.03677250 \\
C & 4.71394497 & 4.62910712 & 4.74324210 \\
C & 1.27157003 & 2.96684688 & -4.74324110 \\
C & 2.29665580 & 3.25211573 & 5.03677350 \\
H & 0.85842130 & 2.58216773 & 0.25917293 \\
H & 5.12709370 & 5.01378627 & -0.25917193 \\
H & 0.60200773 & 2.51076386 & -2.18562471 \\
H & 1.11451154 & 2.65338742 & 2.70400983 \\
H & 4.87100346 & 4.94256658 & -2.70400883 \\
H & 5.38350727 & 5.08519014 & 2.18562571 \\
H & 1.19695718 & 2.98808577 & -6.84802130 \\
H & 2.65959552 & 3.39512017 & 7.10654218 \\
H & 3.32591948 & 4.20083383 & -7.10654118 \\
H & 4.78855782 & 4.60786823 & 6.84802230 \\
H & 0.34448907 & 2.43878927 & -4.63223774 \\
H & 1.36990024 & 2.72414869 & 5.15088151 \\
H & 4.61561476 & 4.87180531 & -5.15088051 \\
H & 5.64102593 & 5.15716473 & 4.63223874 \\
& & & \\
\hline
\end{tabular}

* Electronic address: egh4@slac.stanford.edu

1 H. Koch, O. Christiansen, R. Kobayashi, P. Jørgensen, and T. Helgaker, A direct atomic orbital driven implementation of the coupled cluster singles and doubles (CCSD) model, The Journal of Chemical Physics 228, 233 (1994).

2 T. Helgaker, P. Jørgensen, and J. Olsen, Molecular Electronic Structure Theory, Wiley, New York, 2000.

3 E. Epifanovsky, D. Zuev, X. Feng, K. Khistyaev, Y. Shao, and A. I. Krylov, General implementation of the resolution-of-the-identity and cholesky representations of electron repulsion integrals within coupled-cluster and equation-of-motion methods: Theory and benchmarks, The Journal of Chemical Physics 139, 134105 (2013).

4 H.-S. Hu, K. Bhaskaran-Nair, E. Aprá, N. Govind, and K. Kowalski, Toward enabling largescale open-shell equation-of-motion coupled cluster calculations: Triplet states of $\beta$-carotene, The Journal of Physical Chemistry A 118, 9087 (2014). 